\title{
La peinture des donateurs du temple de Maitreya en Mongolie méridionale $^{\mathrm{i}}$
}

\author{
Isabelle Charleux \\ CNRS, Groupe Religions, Sociétés, Laïcités \\ Paris, France
}

A la fin du XVIe siècle, la tribu mongole orientale des Tümed, établie au nord-est de la boucle du fleuve Jaune, se convertit au bouddhisme tibétain et, à la suite de la fondation en 1572 d'une ville murée, Kökeqota (actuelle Huhehaote ${ }^{(1)}$ ou Hohhot $^{\mathrm{ii}}$ ), entreprend une importante campagne de constructions religieuses : ce sont là les débuts de l'art religieux monumental mongol. Parmi la vingtaine de temples et monastères construits dans l'ensemble du monde mongol de 1575 à 1634 -date de la soumission aux Mandchous ${ }^{\mathrm{iii}}$ de la plupart des tribus de Mongolie méridionale-, le Mayidari-yin juu, « temple de Maitreya », apparaît comme le plus riche, tant par son architecture que par son décor mural ${ }^{\mathrm{iv}}$.

\section{Fig. 1. Vue latérale du temple principal depuis l'ouest (@ Isabelle Charleux)}

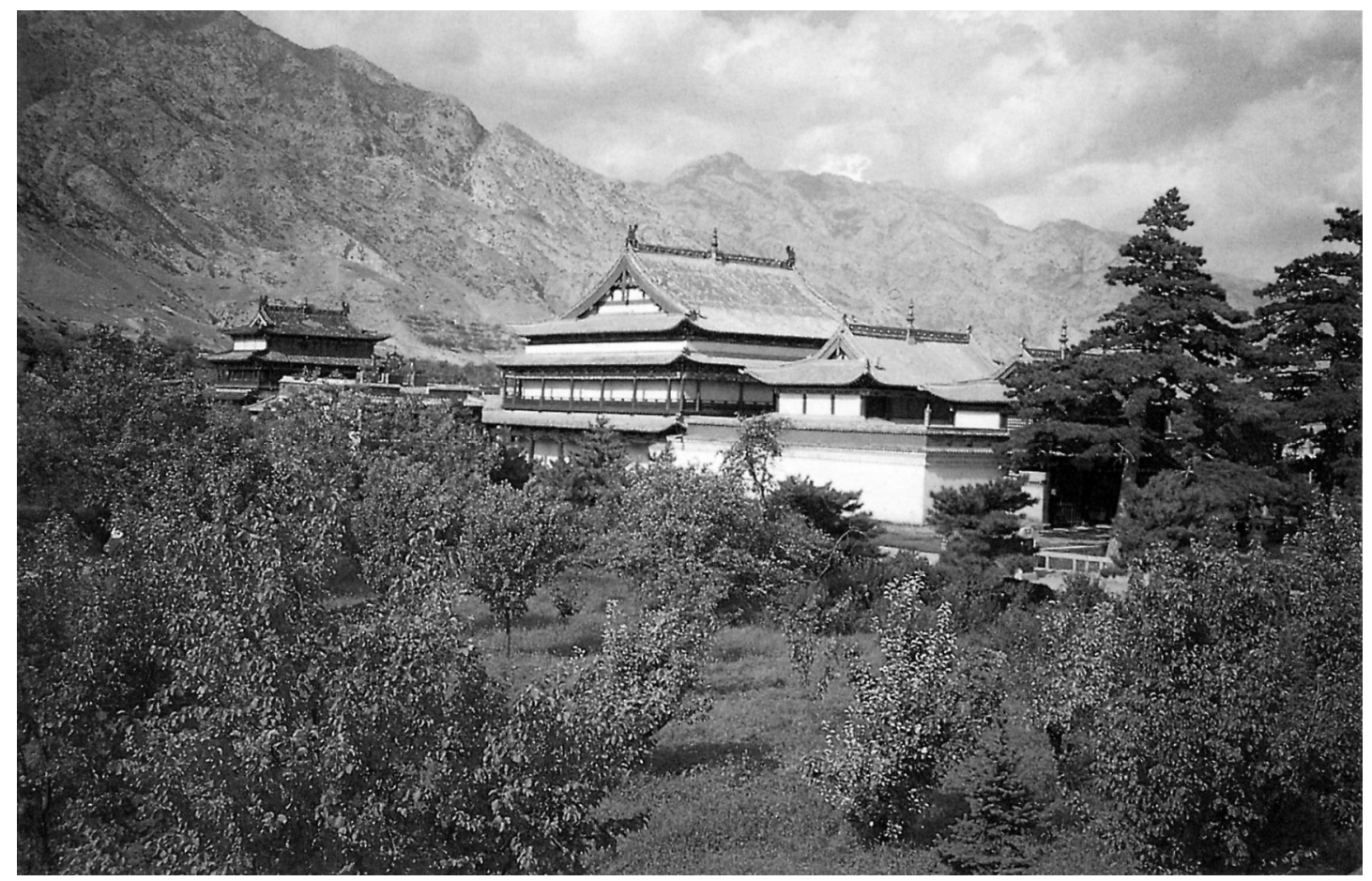


Le Mayidari-yin juu est fondé par le roi tümed Altan qan (1508-1582), descendant d'une branche cadette de la famille gengiskhanide et principal acteur, tant pour des raisons politiques que personnelles, de la réintroduction du bouddhisme en terre mongole dans la deuxième moitié du XVIe siècle ${ }^{\mathrm{v}}$. Sa construction date probablement de 1575 : il s'agit certainement du premier temple construit de cette « renaissance » du bouddhisme -d'autres temples, en effet, étaient itinérants. Situé à 70 $\mathrm{km}$ à l'ouest de Kökeqota, au pied des monts Qarayuna (chinois Daqing shan ${ }^{(2)}$ ), le temple occupe peut-être l'emplacement de l'ancien campement, voire même de la première capitale construite d'Altan qan, antérieure à Kökeqota ${ }^{\mathrm{vi}}$. Les Tümed ont certainement chargé les architectes et charpentiers chinois, prisonniers ou volontairement immigrés au nord de la muraille, d'en édifier les principaux bâtiments.

C'est surtout après la mort d'Altan qan en 1582 que le temple prend une importance politique et religieuse. La troisième femme d'Altan qan (fig. 2), la puissante Noyancu Jünggen (ou Jönggin) qatun (Sanniangzi ${ }^{(3)}$ comme l'appellent les sources chinoises, ca. 1550-1612), conserve, grâce au lévirat ${ }^{\mathrm{vii}}$, le pouvoir pendant trente ans et maintient des relations pacifiques avec la Chine des Ming. Elle habite alors dans l'enceinte du temple de Maitreya, nouveau siège du pouvoir tümed. Une inscription lapidaire placée au-dessus de la porte de l'enceinte fait part d'une importante

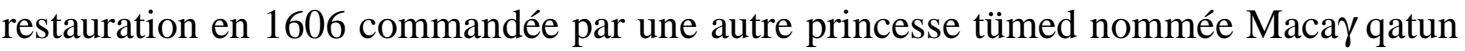
(également connue sous le nom de Cecen tayiqu Uran beyiji ou Baya beyiji, fig. 2), et de la consécration du temple par un lama tibétain réincarné ayant pour titre Mayidari quturtu (1592-1635) ${ }^{\text {viii }}$. A la suite de l'élimination des descendants d'Altan qan en 1640 par les Mandchous, le temple a probablement été désaffecté, et malgré quelques restaurations sous Kangxi (1662-1722) et au XX' siècle, son architecture n'a sans doute pas subi de grandes modifications depuis 1606, contrairement aux autres monastères de la région. Le Mayidari-yin juu est donc un témoin unique de l'architecture et de la peinture murale mongole à la fin des Ming.

Le temple de Maitreya est entouré d'une enceinte fortifiée à peu près carrée, ouvrant par une porte monumentale unique au sud. Couvrant une superficie d'environ $30.000 \mathrm{~m}^{2}$, il comprend aujourd'hui une quinzaine de bâtiments qui s'organisent librement autour de l'édifice principal, le Grand temple ( oul co $\gamma$ cin [fig. 1]) ${ }^{\text {ix }}$. Celui-ci est constitué d'une salle d'assemblée au sud, prolongée par un sanctuaire au nord dont les quatre murs sont recouverts de peintures murales sur $10 \mathrm{~m}$ de hauteur. Les peintures sont divisées en deux registres : la partie supérieure (haute de $7 \mathrm{~m}$ ) représente une figure centrale du bouddhisme tibétain entourée de scènes narratives ${ }^{\mathrm{x}}$. La partie inférieure des murs sud, est et nord (haute de $2 \mathrm{~m}$ ) montre des figures du panthéon bouddhique tibéto-mongol (des dharmapâla, des lokapâla, des arhat et des bodhisattva). Enfin, le registre inférieur du mur ouest figure les principaux acteurs des restaurations et constructions de 1606 [fig. 3, 4]. Cette dernière peinture mérite une attention particulière dans la mesure où il s'agit de la seule peinture murale mongole connue représentant un sujet historique, les divinités du panthéon bouddhique ayant largement dominé l'art mongol jusqu'au $\mathrm{XX}^{\mathrm{e}}$ siècle.

La «peinture des donateurs » du Mayidari-yin juu a déjà fait l'objet de plusieurs études en chinois par Jin Shen ${ }^{(4)}$, Cheng Xuguang(5) et Liu Yibin ${ }^{(6)}$ en $1984^{\text {xi }}$ suite à une campagne de réparation des bâtiments, mais n'a jamais été reproduite dans son intégralité, et aucun spécialiste n'a encore apporté de solution définitive aux différents 
problèmes d'interprétation et de datation qu'elle soulève. La peinture a probablement été restaurée sous les Qing, suite à un incendie datant des années 1632-1634. Le registre supérieur des murs, quant à lui, ne semble pas avoir été repeint. L'on a constaté en effet que la partie inférieure des quatre murs comprend une couche de peinture supplémentaire, d'où une différence de niveau avec la partie supérieure, d'un style légèrement différent ${ }^{\mathrm{xii}}$. Les deux registres sont séparés par une étroite bande grise, et seuls les trônes de Tsong-kha-pa et de Çâkyamuni forment la transition entre les scènes $\mathrm{du}$ haut et du bas [fig. 5]. Dans la partie inférieure, le traitement du paysage est plus chargé, les traits perdent en finesse et les couleurs sont moins lumineuses que dans la partie supérieure. Curieusement, le haut du registre inférieur semble tronqué. D'après Jin Shen ${ }^{(4)}$, l'on a restauré sous les Qing la partie inférieure des quatre murs, après application d'un nouvel enduit, en respectant parfaitement le programme originel. Il faudra attendre une nouvelle étude lors d'une prochaine restauration des peintures pour élucider sur des critères scientifiques la question de la datation.

En tenant compte des hypothèses des savants chinois, nous tentons ici de proposer une interprétation correspondant au contexte politique et religieux de l'époque en nous appuyant sur des découvertes récentes. Après une description succincte, nous étudierons en détail l'apparence extérieure des personnages représentés, et nous proposerons une identification des principaux personnages, pour enfin analyser le style de la peinture.

Fig. 2. Diagramme illustrant la généalogie des descendants d'Altan qan, et les mariages successifs de Jünggen qatun (la Sanniangzi(3), la “Troisième femme ») et de Macay qatun (Baya beyiji). Dessiné par Françoise Aubin (in Serruys, 1987, (C) Variorum Reprints.)

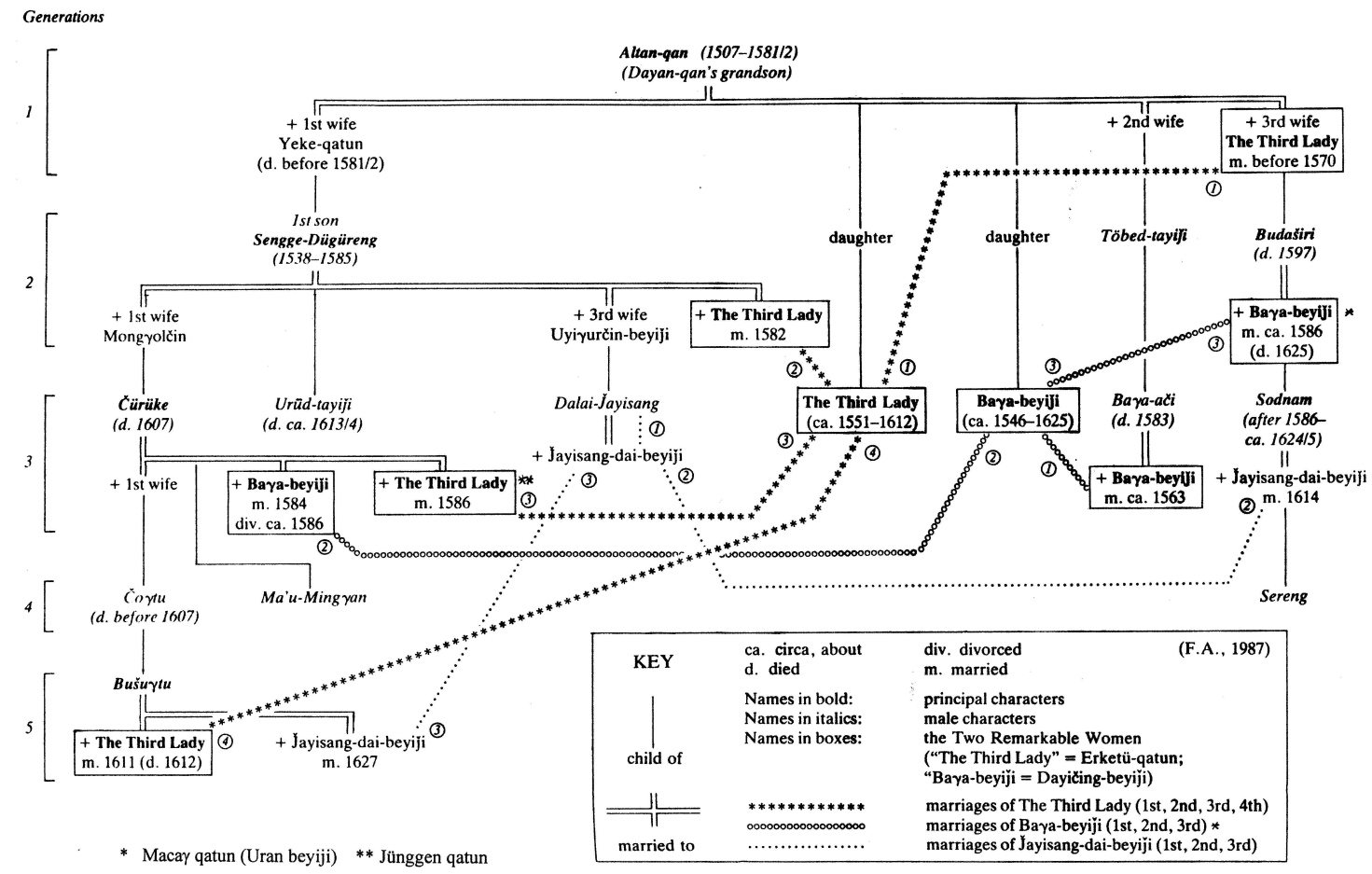




\section{DESCRIPTION DE LA PEINTURE}

La peinture des donateurs, longue de $16 \mathrm{~m}$ et haute de $2 \mathrm{~m}$, est divisée en deux par la base du trône sumeru de Tsong-kha-pa. Celui-ci est orné de vajra, de perles, d'un symbole de longévité et de motifs de brocart d'origine chinoise, et occupe le centre de la composition [fig. 4]. L'ensemble de la peinture est bordée dans sa partie inférieure d'un bandeau de vajra ponctué de motifs spiralés. L'enduit se révèle sous quelques parties abîmées, mais, dans l'ensemble, la peinture est bien conservée. Les couleurs, à base de vermillon, de cinabre, d'ocre et de malachite, sont appliquées en larges aplats ${ }^{\text {xiii }}$. Les tons chauds des vêtements, des éléments de détails, et le rosé des visages s'harmonisent avec le fond vert.

Les personnages représentés, deux femmes et deux hommes de grandes dimensions ( $80 \mathrm{~cm}$ à $1,20 \mathrm{~m}$ de haut), quatre autres personnages principaux, 54 figures de taille réduite et deux divinités bouddhiques, sont tous individualisés dans leur position et leur accoutrement. La plupart sont assis en padmâsana, dans une attitude dévote, entourés de joyaux et de symboles de bon augure sur fond de paysage de montagnes et de cours d'eau. Les deux scènes sont centrées sur une femme (à droite) et sur un homme et une femme qui se font face (à gauche), ceux-ci étant mis en valeur par la place qu'ils occupent dans la composition, et parce qu'ils constituent des pôles vers lesquels sont tournés tous les autres personnages. L'éclairage est uniforme et la composition reste très libre, jouant sur la hauteur des personnages et sur les différents plans.

Une femme âgée, assise sur un meuble de bois sculpté, occupe le centre de la composition de droite [fig. 6]. Elle porte un cafetan jaune ou vert pâle par-dessus une robe rouge orangé, et un chapeau bordé de fourrure. Ses deux nattes sont recouvertes d'une pièce de brocart ; elle tient un chapelet, un vase au creux de la main droite, et un cintâmani, le joyau qui exauce les désirs, dans la main gauche. Deux moines de petite taille, en robe rouge, se tiennent à ses côtés. A gauche, un homme à la barbe fournie est tourné vers elle, dans une attitude de déférence [fig. 7]. Il porte un costume bleu-gris à col de fourrure, et tient un chapelet et un cintâmani. A ses pieds, sur un tapis de feutre, sont alignés quatre personnages, les mains jointes en attitude de prière.

Entre les deux principaux personnages se tient Pishamen ${ }^{(7)}$ (Duo'er tianwang ${ }^{(8)}$ ), forme chinoise de Vaiçravana, en armure de cuivre et casque. Assis sur un trône, il tient une lance-étendart et un arc; ses épaules sont ornées de huit «drapeaux de nuage » (yunqi(9) qui se déploient dans son dos. Son aspect figé dans sa stricte frontalité accentue le contraste avec les donateurs humains, très vivants. A droite de la femme âgée, une jeune femme en robe rouge, tournée vers celle-ci, présente un joyau ; en dessous, quatre jeunes filles jouent de la musique. Deux des instruments sont identifiables : la vielle mongole à deux cordes, qu ur, et la cithare, yatu $\gamma-a$, tous deux d'origine chinoise $\left(e r h u^{(10)}\right.$ et guqin $\left.{ }^{(11)}\right)$. A gauche, quatre moines jouent de la trompette, des cymbales et du tambour, instruments utilisés dans les rituels bouddhiques. A l'extrême droite de la composition se tient le dharmapâla Beg-tse, en armure, entouré de flammes. Il brandit un glaive de la main gauche, dévore un coeur de la main droite, et une lance est posée au creux de son bras.

La scène figurée à gauche du trône sumeru représente une femme relativement jeune, d'une taille identique à celle de la femme plus âgée de la première scène. Cette femme est vêtue d'une longue robe gris-bleu recouverte d'une mante rouge sans manches, et d'une cape jaune qui couvre ses épaules [fig. 8]. Elle porte un chapeau à 
visière multicolore, deux nattes protégées par une pièce de brocart et un imposant collier à pendentifs. Comme la plupart des personnages, elle tient un chapelet et présente un joyau. Tourné vers elle, le personnage en robe monastique rouge a un fin collier de barbe, une moustache effilée et des cheveux longs retombant en boucles [fig. 9]. La visière ondulée de son chapeau reprend le motif du bas de sa robe. D'autres personnages de dimensions réduites remplissent la composition.

Fig. 3. Schéma des peintures du mur ouest du sanctuaire, Mayidari-yin juu. (O Isabelle Charleux)
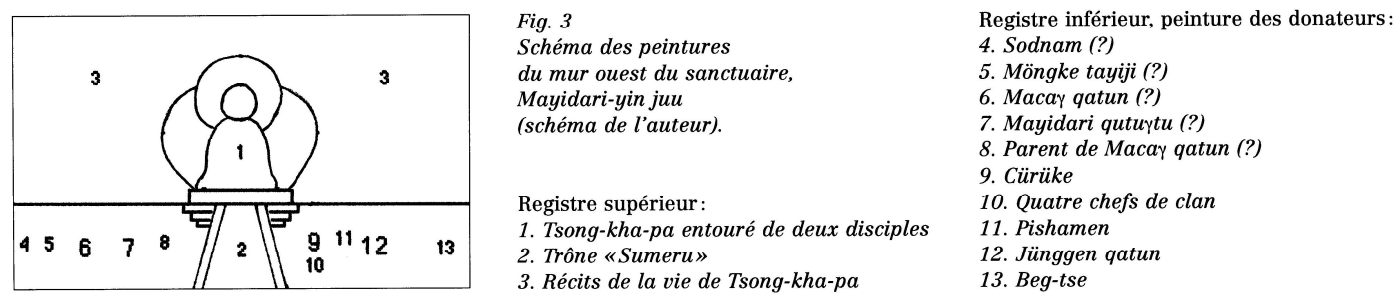

Fig. 4. Dessin de la peinture de donateurs, registre inférieur du mur ouest du sanctuaire. (@) Jin Shen)

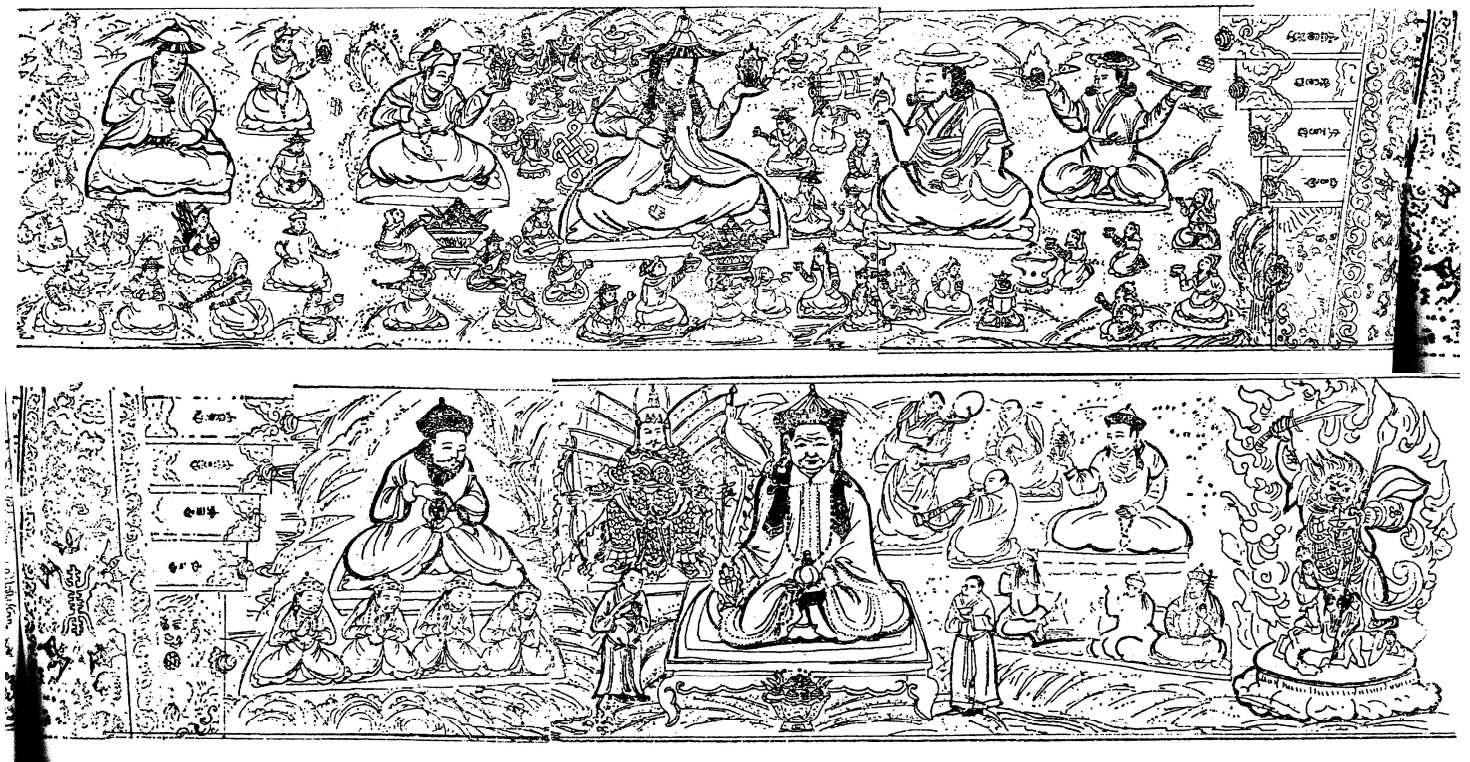

Les personnages semblent flotter à des hauteurs différentes dans le paysage, et sont entourés de très nombreux assistants présentant des offrandes : joyaux, coupes, corail... ou jouant de la musique (pipa, flûte). L'arrière-plan est formé de touches dégradées de vert parsemées de taches brunes et rouges figurant des rochers, de points blancs figurant des fleurs et d'un torrent, le tout composant un paysage verdoyant de montagne et de cours d'eau très éloigné de la steppe aride de Mongolie : il faut certainement y reconnaître la plaine fertile des Tümed (Bayan tala, "plaine riche ») et les monts Qarayuna qui s'élèvent juste au nord du monastère. L'espace entre les personnages est 
rempli par des offrandes disposées dans des corbeilles, des sûtra ou des peintures enroulées qui semblent flotter dans la composition, et par les huit symboles de bon augure situés à gauche de la jeune femme.

\section{Fig. 5. Détail de la transition entre les registres inférieur et supérieur du mur ouest du sanctuaire. (C) Jin Shen)}

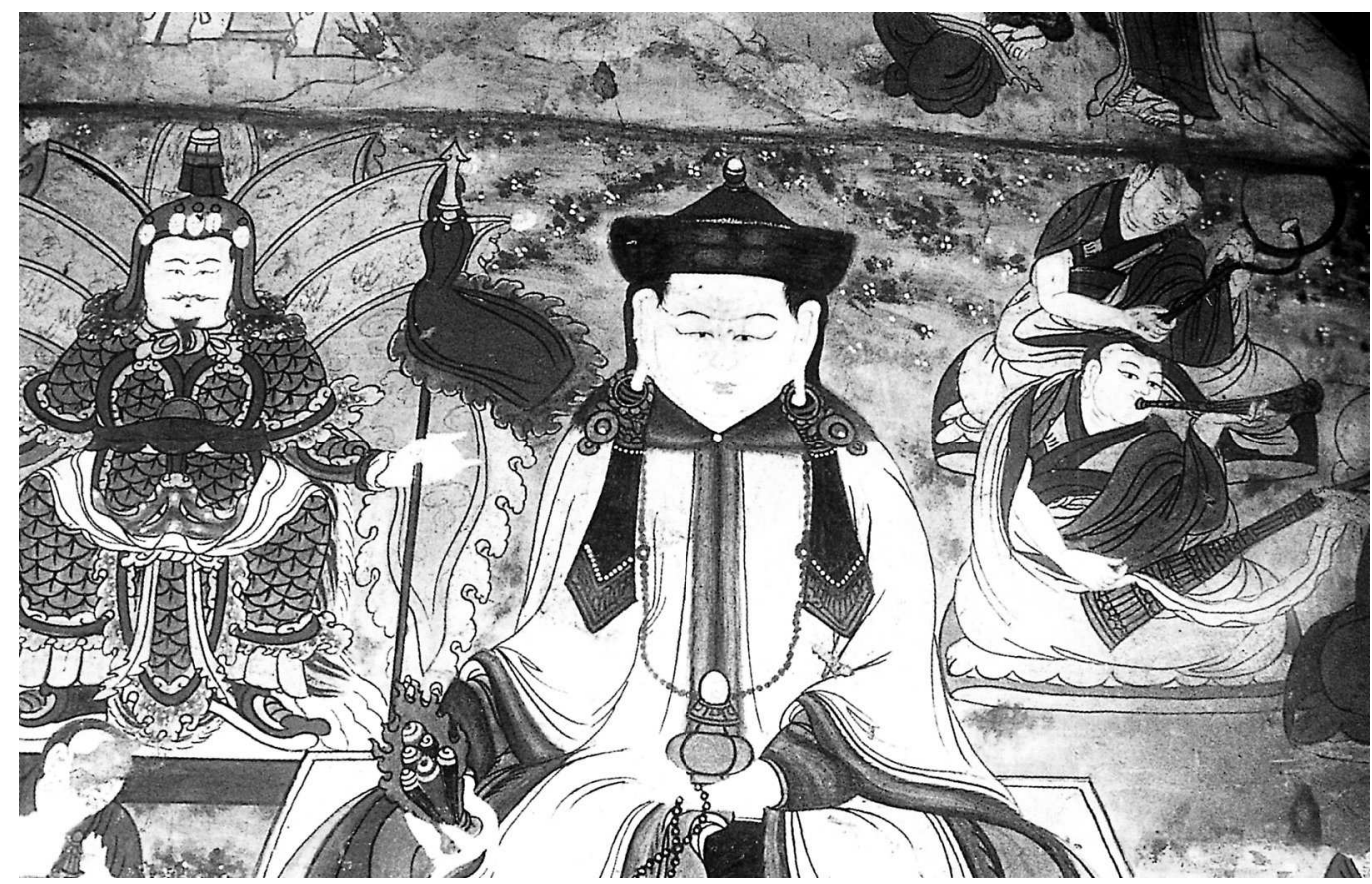

\section{COSTUME DES PERSONNAGES REPRESENTES}

La grande variété des vêtements, des coiffures et des chapeaux mériterait une étude spécifique. La documentation disponible, cependant, est inégale et lacunaire. La plupart des vêtements mongols conservés dans les collections ethnographiques (notamment au Danemark et en Mongolie septentrionale ${ }^{\mathrm{xiv}}$ ) datent de la période Qing, pendant laquelle le costume s'est considérablement simplifié et sinisé, pour aboutir à l'actuel debel, copie du modèle chinois contemporain, boutonné à droite, un peu plus court chez les hommes, long chez les femmes ${ }^{\mathrm{xv}}$. Il faut donc, comme tente de le faire Jin Shen $^{(4)}$ dans son analyse, comparer les costumes de la peinture à ce que l'on connaît du vêtement sous les Yuan et sous les Ming par les rares sources écrites et iconographiques, afin de rassembler quelques éléments permettant de comprendre les relations entre les différents personnages et de les identifier.

Le costume mongol varie selon le sexe, la tribu, l'époque et le rang social. Les coutumes vestimentaires des Mongols de l'époque Ming ne nous sont connues que par des sources littéraires chinoises -essentiellement le Beilu fengsu ${ }^{(12)}$ [ "Coutumes des barbares septentrionaux »], rédigé en $1594^{\mathrm{xvi}}$-, et il y a de grandes chances pour que leurs descriptions se rapportent précisément aux tribus Tümed et Ordos avec lesquelles les Ming entretiennent des relations privilégiées depuis le traité de paix de 1571. La peinture, de même que le Beilu fengsu ${ }^{(12)}$, se situe nettement dans le contexte de la 
noblesse tümed convertie, avec ferveur semble-t-il, au bouddhisme. Comme le souligne le Beilu fengsu ${ }^{(12)}$, «hommes et femmes, jeunes et vieux, sans distinction, ont constamment le rosaire en main, et ils ne le quittent jamais $»{ }^{x v i i}$, ce qui se vérifie sur la peinture.

Fig. 6. Détail de la partie droite : Jünggen qatun. (C Jin Shen)

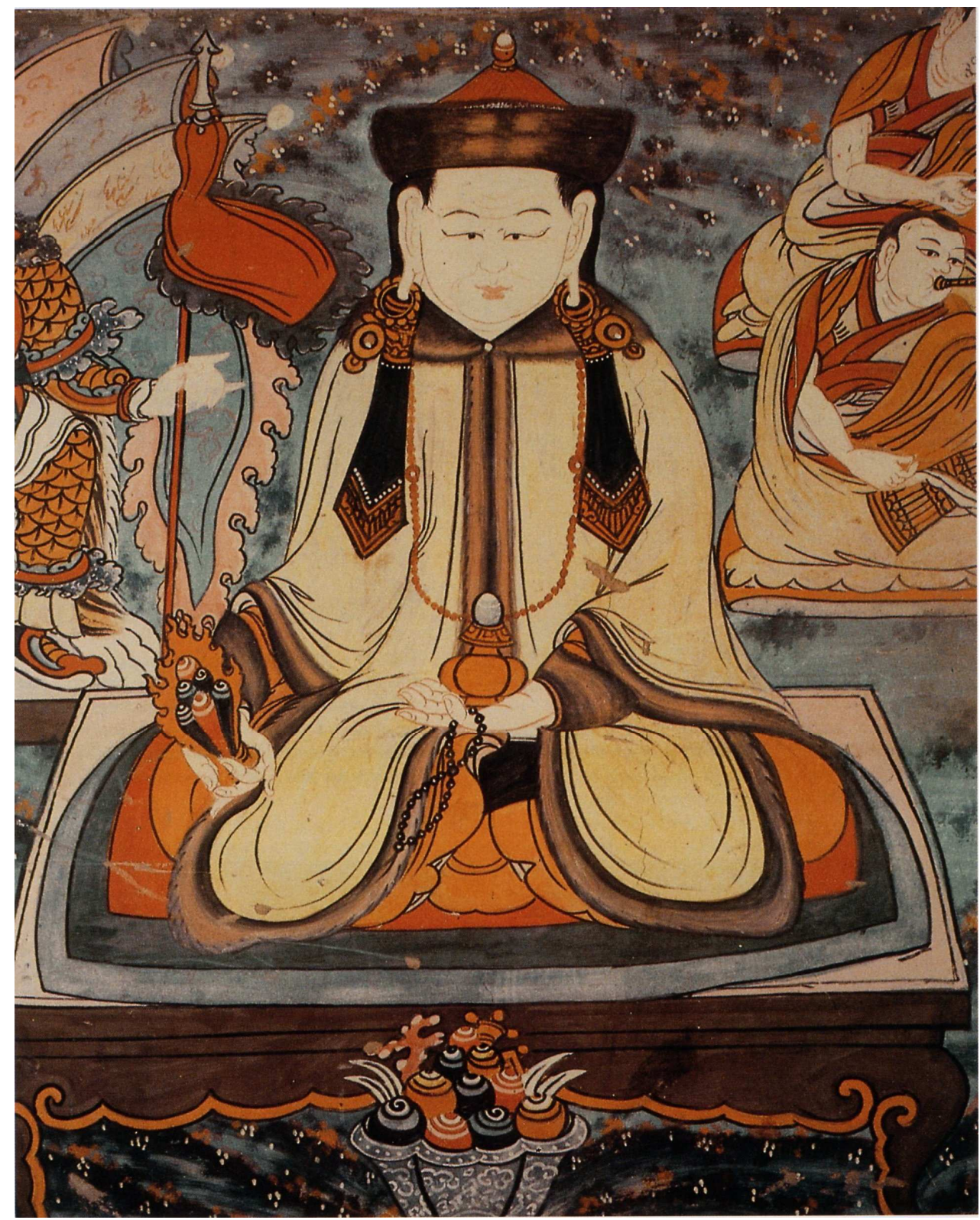

Seuls le lama vêtu de rouge de la partie gauche, l'homme à la robe bleu-gris de la partie droite, et trois des quatre petits personnages en dessous de ce dernier portent la barbe ou la barbiche et la moustache, qui soulignent leur âge et leur statut social. « Tout le monde, jeunes et vieux, se coupe les cheveux. Ils ne laissent que les cheveux sur le derrière de la tête, sur une largeur d'environ un pouce, et ils en tressent une petite natte ${ }^{\text {xviii }}$. Les 
tempes des quatre petits personnages en dessous de l'homme barbu de la partie droite semblent être rasées au-dessus de l'unique mèche (nattée ?) qui retombe sur leur épaule droite. Les gens du commun comme les nobles, qui portaient une ou deux tresses sous les Yuan ${ }^{\text {xix }}$, n'en ont en général qu'une sous les Ming et les Qing, parfois à l'arrière, mais plus fréquemment, comme dans la peinture, derrière les oreilles. Après la première coupe de cheveux à l'âge de trois ans, la natte ne devait jamais être coupée ; elle était un symbole de statut social ${ }^{\mathrm{xx}}$. On a justement retrouvé à l'intérieur du stûpa funéraire abrité par le pavillon appelé Taihou miao ${ }^{(13)}$ («temple de l'Impératrice ») ${ }^{\mathrm{xxi}}$, à l'intérieur de l'enceinte du temple, dix nattes, dont une natte d'homme, de plus de vingt centimètres. L'homme barbu au vêtement gris-bleu à droite ne porte pas de natte, ou peut-être est-elle dans son dos [fig. 7]. Jin Shen ${ }^{(4)}$ émet cependant l'hypothèse qu'il a pu couper sa natte en signe de soumission aux Ming, et adopter les coutumes chinoises, suite au traité de paix signé en $1571^{\text {xxii }}$. Or si les chroniques chinoises perçoivent les accords de 1571 comme la reddition des Tümed, les sources mongoles affirment en revanche que ce sont les Chinois qui payent tribut ${ }^{\mathrm{xxiii}}$. Une troisième hypothèse nous vient à l'esprit : c'est bien en signe de soumission, non aux Chinois, mais à la femme âgée au centre de la partie droite, que sa natte est coupée.

Fig. 7. Détail de la partie droite : Cürüke. (@ Jin Shen)

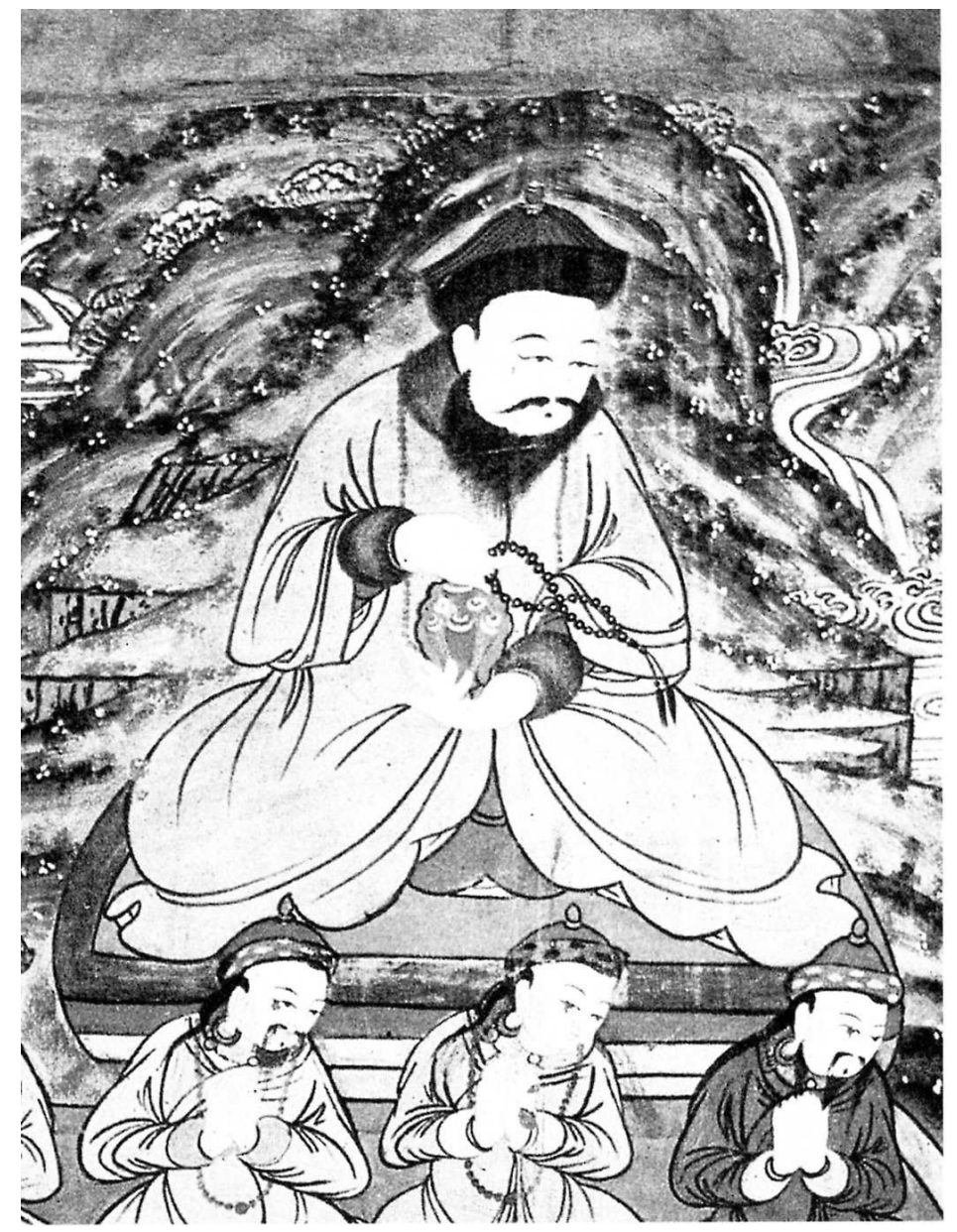


Fig. 8. Détail de la partie gauche : Macarqatun (?) (@ Jin Shen)

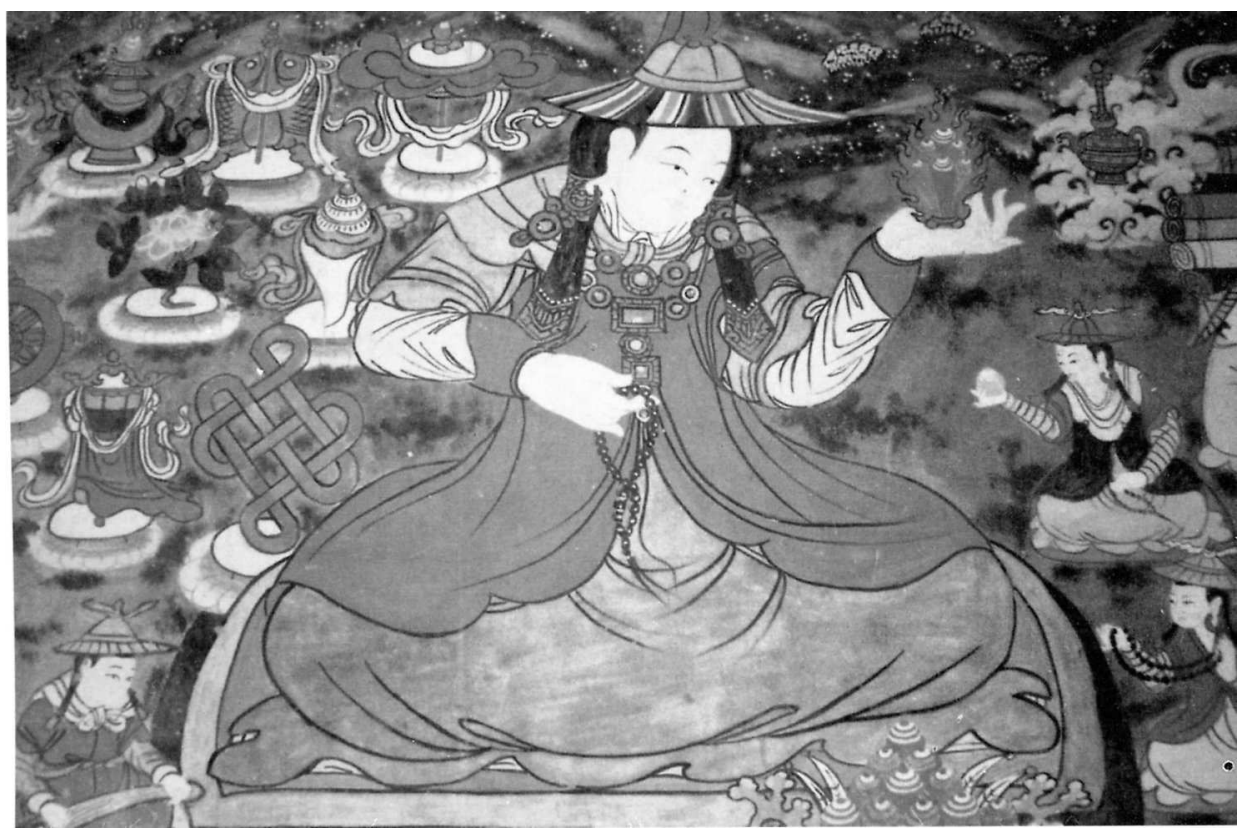

La description faite par le Beilu fengsu ${ }^{(12)}$ de la coiffure féminine correspond à la peinture : pour la chevelure des jeunes filles, « on en tresse une dizaine de petites nattes, qui pendent devant et derrière la tête, ainsi que des deux côtés, et ce n'est qu'au jour de son mariage [...] qu'on lui tresse les cheveux en deux nattes, qui finissent en pelotes, et lui pendent des deux côtés sur les oreilles. Les oreilles sont percées d'un petit trou, où pendent des boucles et des pendants d'oreilles en or ou en argent ${ }^{\text {xxiv }}$. Les deux principaux personnages féminins portent deux nattes protégées par des fourreaux de tissu et portées devant, coutume qui a encore cours en Ordos au $\mathrm{XX}^{\mathrm{e}}$ siècle. Tout à fait à droite de la peinture, quatre femmes qui jouent de la musique et chantent ont divisé leur cheveux en dix tresses ${ }^{\mathrm{xxv}}$ : ce sont donc des jeunes filles non mariées. Ces coutumes, jadis répandues dans toute la Mongolie, se sont maintenues encore très récemment chez les $\operatorname{Ordos}^{\text {xxvi }}$. La femme à droite du lama en rouge porte ses cheveux non attachés, ce qui caractérise les veuves ${ }^{\mathrm{xxvii}}$.

Les ornements et bijoux sont en Mongolie un élément essentiel du costume des hommes comme des femmes ; ils sont particulièrement importants pour les femmes mariées dont ils constituent à la fois le symbole du statut social et une partie de la dot. La jeune femme qui occupe le centre de la partie gauche porte un lourd collier à pendentifs rectangulaires et circulaires qui pourrait, d'après la couleur, être en or serti de turquoise ou en argent [fig. 8]. Les quatre hommes représentés en dessous de l'homme au vêtement gris-bleu portent de grandes boucles d'oreille ${ }^{\text {xxviii }}$. On remarque que les boucles d'oreille portées par les femmes sont proches de celles des bodhisattva peints dans la partie inférieure du mur sud. Ces bijoux ne ressemblent pas, en revanche, aux pendants que l'on voit dans les peintures des tombes Yuan aux oreilles des hommes comme des femmes $^{\text {xxix }}$. Enfin les coiffes ne comportent pas les riches bijoux et surtout les complexes parures de tête réalisées en argent et serties de turquoise et de corail, pendant sur chaque joue ou sur le front, que portent les femmes de nombreuses tribus sous les Qing ${ }^{\mathrm{xxx}}$. Les Mongols Tümed portent-ils moins de bijoux que les tribus dont 
nous connaissons le costume sous les Qing ? Peut-être la peinture tümed veut-elle plutôt délibérément représenter le costume quotidien de donateurs qui offrent leurs trésors à l'église bouddhique.

Tous les laïcs portent un chapeau : on en observe huit variétés, en feutre et/ou en paille tressée avec des rebords à fourrure. D'après le Beilu fengs $u^{(12)}$, « on fabrique ces chapeaux avec du feutre, des peaux, ou de la paille de froment, qu'on tresse et qu'on enroule tout autour, pour avoir la forme du chapeau [...] Hommes et femmes portent ces chapeaux » ${ }^{\mathrm{xxi}}$. Le chapeau de paille tressée et de fourrure surmonté d'une pierre précieuse rouge, porté par la femme âgée, et sa variante portée par l'homme barbu à sa gauche, sont des chapeaux d'hiver courants dans toute la Mongolie ${ }^{\text {xxii }}$. Jin Shen ${ }^{(4)}$ les identifie au chapeau rond des Yuan ${ }^{\text {xxxii }}$ qu'avait interdit le premier empereur Ming (Hongwu, 1368-1399) ${ }^{\mathrm{xxxiv}}$. S'agit-il du petit chapeau que décrit le Beilu fengsu ${ }^{(12)}$ : « on y attache une ficelle qui passe derrière la nuque ; le bord du chapeau est très étroit et ils attachent une pierre précieuse rouge sur le sommet et une image de Bouddha en argent sur la partie antérieure ${ }^{\mathrm{xxx}}$ ? L'on ne voit toutefois ici aucune figuration de Bouddha.

La coiffure la plus représentée, portée entre autres par la jeune femme de la partie gauche et par l'homme de l'extrême gauche, est un chapeau d'été en paille tressée à visière multicolore, surmonté d'une sorte de pompon (ximao $\left.{ }^{(14)}\right)$. Il était également porté sous les Yuan mais a peut-être une origine chinoise, comme le suggère Jin Shen ${ }^{(4)^{x x x v i}}$. A gauche de la jeune femme, un jeune homme porte un huntuomao ${ }^{(15)}:$ c'est un chapeau en laine et feutre, à deux revers latéraux s'interrompant en pointes, connu dès l'époque Tang ${ }^{\mathrm{xxxvii}}$. On y voit encore le nuanmao ${ }^{(16)}$, chapeau chaud pour se protéger du vent, porté sous les Yuan ${ }^{\text {xxxviii }}$. Il est ici un peu modifié : le rebord est moins large, mais peut être rabattu pour faire de l'ombre. Derrière le lama en rouge, une femme porte un chapeau «en forme de fleur de lotus ${ }^{\text {xxxix }}$. La peinture de donateurs figure donc des couvre-chefs mongols déjà portés sous les Yuan, dont le sommet est orné d'une perle ou d'un noeud de soie en fonction du rang de noblesse. Certains seront remis à la mode par les Mandchous ${ }^{\mathrm{xl}}$. L'on ne voit pas ici le célèbre bo tra (ch. $\left.g u g u^{(17)}\right)$ porté par les femmes mariées sous les Yuan, échafaudage haut d'un mètre environ autour d'un squelette en bois peint, entouré de soie : il semble avoir été rarement porté sous les Ming ${ }^{\text {xli }}$.

L'adéquation de la peinture des donateurs à ce que l'on connaît des coutumes tümed au XVI $\mathrm{I}^{\mathrm{e}}$ siècle s'observe aussi dans le vêtement. Les laïcs portent un cafetan par-dessus une robe d'été ou d'hiver (urtu camca, «habit long ») dont les modèles, les coupes et les couleurs sont très variés. Le cafetan gris-bleu de l'homme barbu est simple, sans décor et sans ceinture ; il s'ouvre sur le devant, sans boutonnière apparente et les manches, au-dessus des poignets, sont larges. Les quatre personnages situés en dessous portent un vêtement quasi identique, mais à pans croisés et fermé à droite ${ }^{\text {xlii }}$. Jin Shen ${ }^{(4)}$ le rapproche des zhisunfu(18), costumes d'apparat sous les Yuan, qu'Altan qan portait peut-être lorsqu'il rencontra le Dalai lama ${ }^{\text {xliii }}$. La plupart des vêtements d'hiver ont ici un col de fourrure, innovation qui semble dater des Ming (les cafetans d'époque Yuan n'ont pas de col, ou bien un col rond ou croisé, sans fourrure $)^{\text {xliv }}$. La femme âgée porte un cafetan aux manches très larges dont le col de fourrure retombe sur les épaules, à moins qu'il ne s'agisse d'une cape fermée devant par le col de fourrure comme le pense Jin Shen ${ }^{(4)^{x l v}}$. Ces cafetans ont en général des manches larges et courtes, arrivant au-dessus des poignets, du coude ou au niveau des épaules ; au contraire le vêtement de dessous est à manches longues, dont les poignets étroits sont agrémentés de fourrure en hiver ; il 
s'attache à droite ${ }^{\text {xlvi }}$. Ces deux costumes, conçus pour chevaucher et tirer à l'arc avec aisance, n'ont apparemment pas beaucoup changé depuis les Yuan ${ }^{\text {xlvii }}$.

Fig. 9. Détail de la partie gauche : le Mayidari qutułtu (?).(@ Jin Shen)

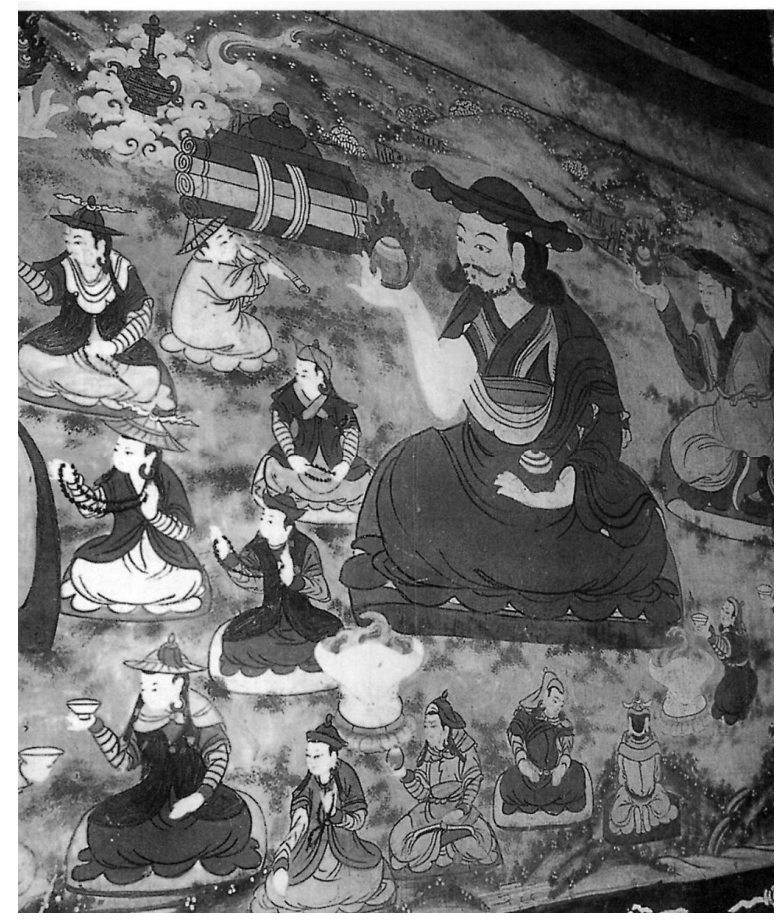

Fig. 10. Jambhala, registre inférieur du mur intérieur sud de l'entrée du sanctuaire. (@) Jin Shen)

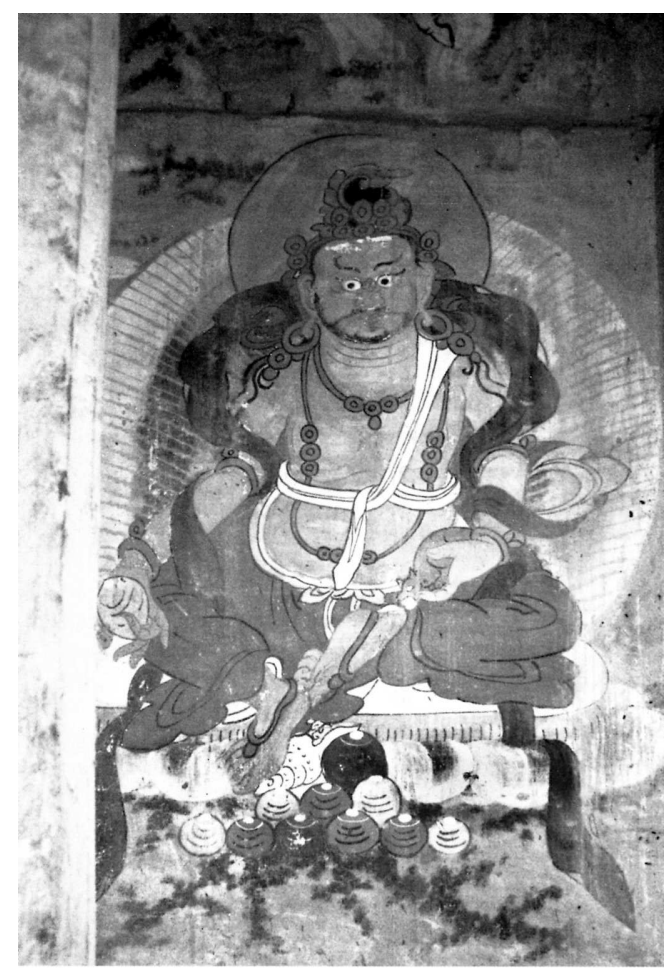


Dans la partie gauche de la peinture, les personnages portent des vêtements d'été, qui diffèrent des habits d'hiver par l'épaisseur, le col et la couleur. Les manches de la robe sont retroussées, découvrant le revers de couleur différente. La jeune femme à gauche porte une longue mante ouverte, sans col et sans manches, tombant jusqu'aux genoux : il s'agit certainement du uжiji (a miji, uuji $)^{\text {xlviii }}$, que les textes chinois appellent bijia $^{(19)}$, également pratique pour monter à cheval et tirer à l'arc ${ }^{x l i x}$. Cette mante semble avoir été à la mode sous les Ming pour des femmes de tous âges : on en trouve treize ou quatorze dans la peinture. On voit encore des cafetans de dessus aux manches resserrées aux poignets, avec ou sans col, et des robes de dessous à motifs de rayures.

Tous les laïcs sont assis en padmâsana et les bords de leurs cafetans retombent en vagues, motif rappelant les pétales de lotus servant de base aux divinités bouddhiques. Le pantalon porté en dessous de la robe et les bottes ne sont pas visibles. Les femmes ne portent pas de ceinture, ce qui est le cas dans de nombreuses tribus ${ }^{1}$. Les hommes en portent une permettant à la partie supérieure du vêtement de former une large poche. La ceinture est un élément d'une grande importance dans le costume masculin, car elle est le siège de l'âme de la personne. Dans L'Histoire secrète des Mongols, Gengis Khan suspend sa ceinture à son cou et ôte son chapeau lorsqu'il rend hommage au Ciel : c'est un signe de soumission envers un supérieur ${ }^{\text {li }}$. Si tous les hommes de la partie gauche portent une ceinture, ce n'est pas le cas de ceux de la partie droite. La scène de droite se passant en hiver, il est très probable que le manteau d'hiver des hommes cache leur ceinture. L'homme situé à l'extrême gauche a revêtu un gilet qui cache en partie sa ceinture. L'homme barbu, à droite, a pu ôter sa ceinture et couper sa natte en signe de soumission, ou encore d'appartenance au clergé [fig. 7].

Le cafetan de la femme âgée est d'un vert très pâle, proche du jaune, de la même couleur que la toge $\mathrm{e}^{\text {lii }} \mathrm{du}$ moine à sa droite ; de plus sa robe est rouge-orangé. Les autres vêtements des laïcs adoptent des couleurs variées à l'exception du jaune, couleur sacrée, strictement réservée aux moines (ceux-ci portent également du rouge, de l'orange et du brun) ${ }^{\text {liii }}$ : la donatrice principale serait-elle ainsi assimilée à une religieuse ?

Par rapport aux vêtements portés sous les Qing, décorés de brocarts, de nombreux motifs chinois (dragon, caractère $f u^{(20)}$ [« bonheur »] symbolisé, noeud sans fin...), l'on est surpris ici de l'absence totale de décor de ces robes et cafetans, à l'exception des vêtements de dessous à rayures. La soie, le brocart, les motifs de bon augure n'étaient pratiquement pas utilisés sous les Yuan ${ }^{\text {liv }}$, bien que l'on ait retrouvé des vêtements funéraires richement ornés ${ }^{\mathrm{lv}}$. En revanche, d'après le Beilu fengsu ${ }^{(12)}$, les Mongols aiment les tissus à fleurs et ont pris goût à la soie ${ }^{\text {lvi }}$. La peinture est trop détaillée pour que l'on puisse soupçonner le peintre d'avoir omis ces motifs; on peut cependant rappeler qu'à la fin des Ming les Mongols manquent de tissu, au point qu'ils n'hésitent pas à en dépouiller les Chinois qu'ils capturent ${ }^{\text {lvii }}$. Les somptueuses robes de soie offertes par la cour chinoise, comme la robe rouge brodée de dragons donnée par l'empereur à Altan qan en 1571, ne sont visiblement portées qu'à l'occasion des relations diplomatiques sino-mongoles ${ }^{\text {lviii }}$. La peinture des donateurs tend, semble-t-il, à montrer les Tümed dans leur tenue quotidienne et non dans un habit d'apparat offert par les Chinois.

Les moines de la peinture sont vêtus, comme les moines tibétains, d'un gilet sans manche, rouge et jaune, porté à même la peau et croisé devant, d'une longue jupe et d'un châle ou toge jaune ${ }^{\text {lix }}$. Le lama barbu, en face de la jeune femme, a une toge rouge et un chapeau de la même couleur. Le dieu Pishamen ${ }^{(7)}$ porte une armure identique à ce que 
l'on peut voir sur les peintures d'époque Ming, et surtout aux armures chinoises et mongoles contemporaines ${ }^{\mathrm{lx}}$. Seuls les « drapeaux de nuages », dans son dos, ne font pas partie de l'iconographie traditionnelle de Pishamen ${ }^{(7)}$ en Chine : ils caractérisent les costumes de généraux de l'opéra chinois. Le peintre du temple de Maitreya a pu s'inspirer de l'imagerie populaire chinoise, qui représente fréquemment des compositions théâtralisées ${ }^{\mathrm{lxi}}$.

\section{Proposition D’IDENTIFICATION DES PERSONNAGES}

L'analyse formelle de la peinture permet globalement de confirmer la date de l'événement représenté -la fin des Ming-, tout en mettant en valeur sa spécificité dans la simplicité vestimentaire des personnages, et dans le fait que chacun est soigneusement individualisé par son accoutrement et son attitude. Bien qu'aucune inscription ni aucune autre représentation contemporaine ne permette de les identifier avec certitude, les chroniques chinoises et la stèle relatant les restaurations de 1606 permettent de formuler des hypothèses quant à leur identité.

Les deux personnages principaux par leur taille et leur position sont deux femmes d'âges différents. Les auteurs ayant étudié la peinture s'accordent unanimement à identifier la femme âgée qui occupe le centre du panneau de droite à la troisième épouse d'Altan qan, Jünggen qatun, en raison de son âge, de son attitude et de la richesse de son vêtement bordé de fourrure [fig. 6] ${ }^{\text {lxii }}$. Bien qu'elle ne soit pas mentionnée dans l'inscription de 1606, Jünggen qatun est également liée au Mayidari-yin juu, dont elle hérite après la mort d'Altan qan ; il est probable qu'elle a participé aux travaux. On sait qu'il existait des portraits de Jünggen qatun contemporains de la restauration du Mayidari-yin juu : dans le chapitre sur les religions du Wanli yehuo bian ${ }^{(21)}$ écrit en 1606, Shen Defu ${ }^{(22)}$ (1578-1642) fait l'éloge de la piété de Jünggen qatun : « J'ai vu moi-même son visage en portrait : il est rond et plein, avec des cils magnifiques, et ne ressemble pas à celui d'une mongole. Elle porte des perles à son cou, égrène un chapelet de sa main et semble réciter les noms du Bouddha »xiii. Or au Mayidari-yin juu, sa silhouette générale, de face, contrairement aux autres personnages, est caractéristique des représentations de Bouddha, et des représentations « iconiques » en général. Les couleurs de ses vêtements ont une connotation religieuse ; ses oreilles au lobe distendu par les pendants, et qui semblent prolongées par les fourreaux qui protègent ses nattes rappellent les longues oreilles de Çâkyamuni. Le récipient qu' elle tient est semblable au vase rempli de liqueur d'immortalité d'Amitâyus, bodhisattva de longévité. Le vase rempli de joyaux posé à ses pieds semble lui être destiné. A droite et à gauche, deux lamas debout, de petite taille, tiennent un bol. Ils ne portent pas le vêtement monastique tibétain, mais une simple robe longue de couleur orange, croisée et fermée par une ceinture, proche de la robe de l'arhat Dharmatrâta peint sur la partie inférieure du mur nord [fig. 12] : c'est donc une robe étrangère, se voulant peut-être indienne. Jin Shen ${ }^{(4)}$ propose de les identifier à Ânanda et à Kâçyapa, les deux disciples couramment représentés à droite et à gauche de Çâkyamuni $^{\text {lxiv }}$, bien qu'ils ne tiennent pas le sistre qui est leur attribut habituel ; il s'agirait, étant donné leur apparence et suivant la tradition tibétaine, plus probablement de Çâriputra et de Maudgalyâna. Cette représentation religieuse de Jünggen qatun est corroborée par des textes ; on lit dans la biographie d'Altan qan que le Dalai lama reconnaît Jünggen qatun comme l'incarnation d'un bodhisattva ${ }^{\text {lxv }}$; par ailleurs il n'est 
pas rare que des princesses mongoles se considèrent comme l'incarnation d'un bodhisattva et en prennent le nom ${ }^{\text {lxvi }}$. Les sources mongoles mentionnent essentiellement Jünggen qatun dans des contextes religieux, en la nommant incarnation de Târâ verte ${ }^{\text {lxvii }}$. On peut donc affirmer que Jünggen qatun est délibérément représentée en bodhisattva. Ce serait bien en signe de soumission et de respect que les hommes qui sont tournés vers elle en position d'adoration ne portent pas de ceinture, et pas de natte non plus pour le plus important d'entre eux.

L'homme barbu tourné vers Jünggen qatun dans une attitude de déférence est probablement celui avec lequel elle est mariée en 1606, soit son troisième mari, Cürüke, petit-fils d'Altan qan, et qui a alors une quarantaine d'années [fig. 2, 7]. Leurs relations sont réputées très harmonieuses, mais Jünggen qatun, par son passé et sa personnalité, devait conserver sur lui une grande autorité ${ }^{\text {xviii }}$. Ils habitent ensemble à Kökeqota et sont connus pour leur rôle de mécène dans la traduction du $b K a$ '-'gyur en mongol de 1602 à 1607. En dessous de Cürüke, les quatre hommes pourraient être quatre chefs de clan sous son autoritéxix.

Pour Cheng Xuguang(5) et Liu Yibin ${ }^{(6)}$, le panneau de gauche représente Jünggen qatun, plus jeune-la peinture entière lui serait consacrée-, faisant face à Altan qan, son premier époux [fig. 8, 9] ${ }^{\mathrm{lxx}}$. La jeunesse de Jünggen qatun se déroulerait dans une scène estivale, sa vieillesse, dans une scène hivernale. Or les deux personnages de gauche ne semblent pas avoir la cinquantaine d'années de différence qui séparait Altan qan et Jünggen qatun; d'autre part, il semble exclu que l'on ait pu représenter le roi des Tümed en habit monastique, sans attributs royaux et, de plus, dans une position d'infériorité par rapport à sa troisième femme. Nous rejoignons donc l'opinion de Jin Shen ${ }^{(4)}{ }^{1 x x i}$, qui

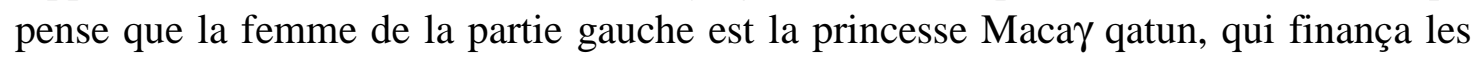
constructions de 1606 et fit fabriquer une statue de Maitreya que consacra la même année le Mayidari quturtu.

Après la mort de son premier mari en 1583, Maca q qatun habite probablement au Mayidari-yin juu, tandis que Jünggen qatun, alors mariée à Sengge Dügüreng, a

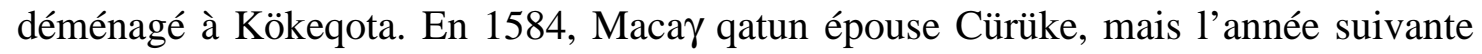
celui-ci se voit contraint d'épouser Jünggen qatun lorsqu'il succède à son père Sengge qui vient de décéder (fig. 2). Ces querelles de succession et de mariage ont divisé la tribu Tümed en deux factions opposées basées l'une à Kökeqota (la faction orientale de Jünggen qatun), l'autre, au Mayidari-yin juu (la faction occidentale de Maca q qatun). Leurs différents semblent cependant apaisés en 1606, ce qui explique que l'on retrouve les deux princesses associées sur la même peinture votive. De même que Jünggen qatun, Maca q qatun se considère comme une incarnation de Târâtxxii : l'incarnation de la Târâ blanche de la partie gauche ferait donc pendant à l'incarnation de la Târâ verte dans la partie droite. Deux problèmes demeurent : en 1606, les deux princesses ont une cinquantaine d'années ${ }^{\text {lxxiii }}$ alors qu'une nette différence d'âge apparaît entre elles dans la peinture, et la différence de saison n'est pas expliquée. Jin Shen ${ }^{(4)}$ pense qu'on a voulu

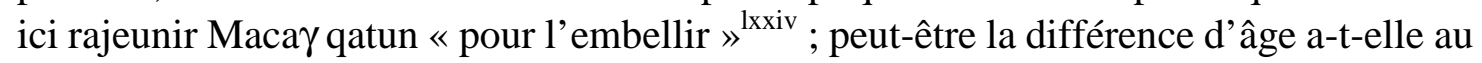
contraire pour but de souligner l'autorité incontestée et la sagesse de Jünggen qatun, épouse de quatre générations de rois. Soulignons le caractère piquant de la scène : Cürüke, représenté auprès de Jünggen qatun, a été le second époux de Maca qatun. En 1606 cette dernière est, selon les sources, soit une des trois femmes de Cürüke, soit divorcée de ce dernier et épouse (en troisième noce) d'un fils de Jünggen qatun (fig. $2)^{\operatorname{lxxv}}$ ! 
Fig. 11. Détail de la partie gauche : Möngke tayiji (?), petit-fils de Jünggen qatun. (C) Jin Shen)

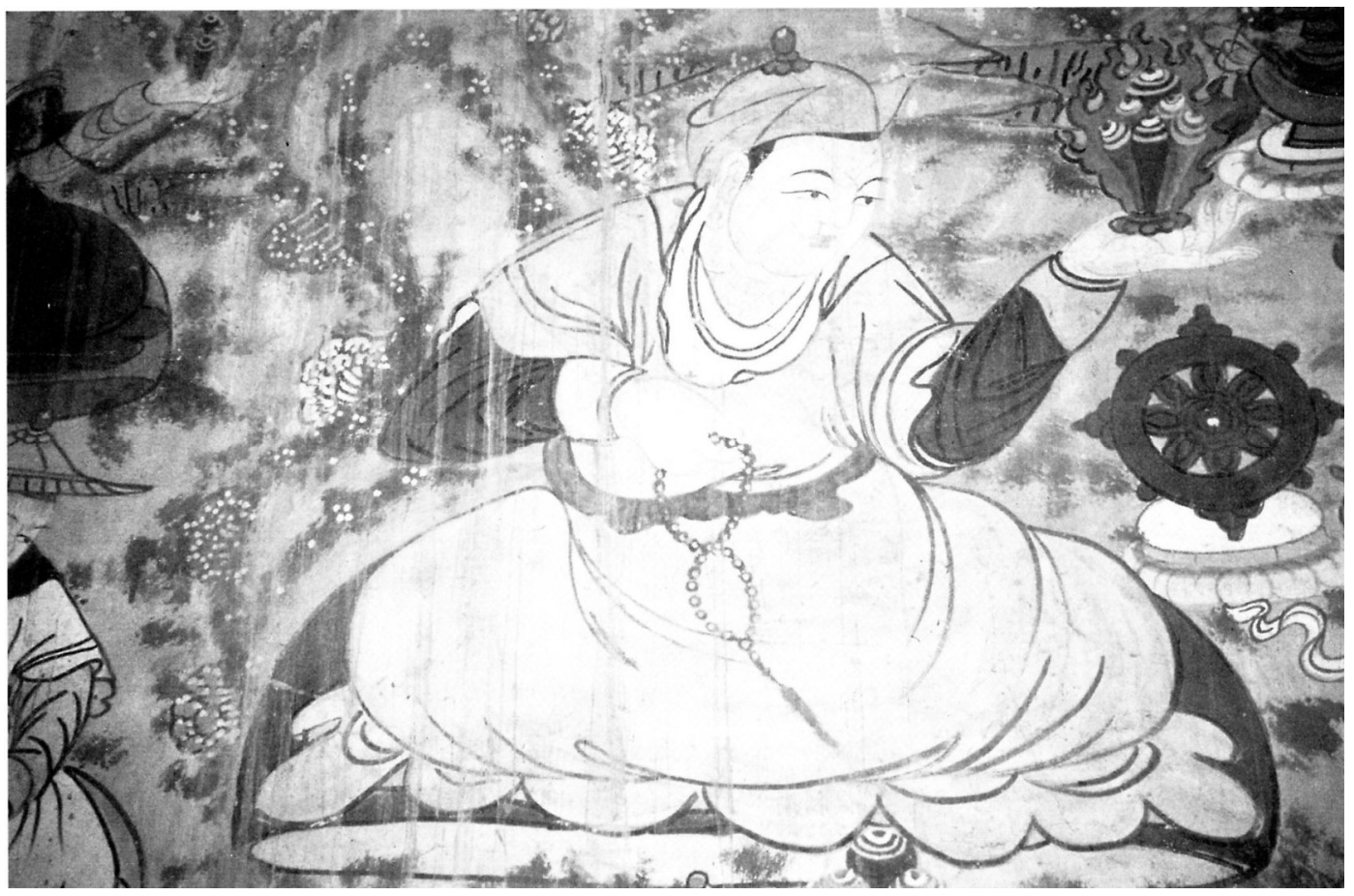

Fig. 12. Dharmatrâta, registre inférieur du mur nord du sanctuaire (@ Jin Shen)

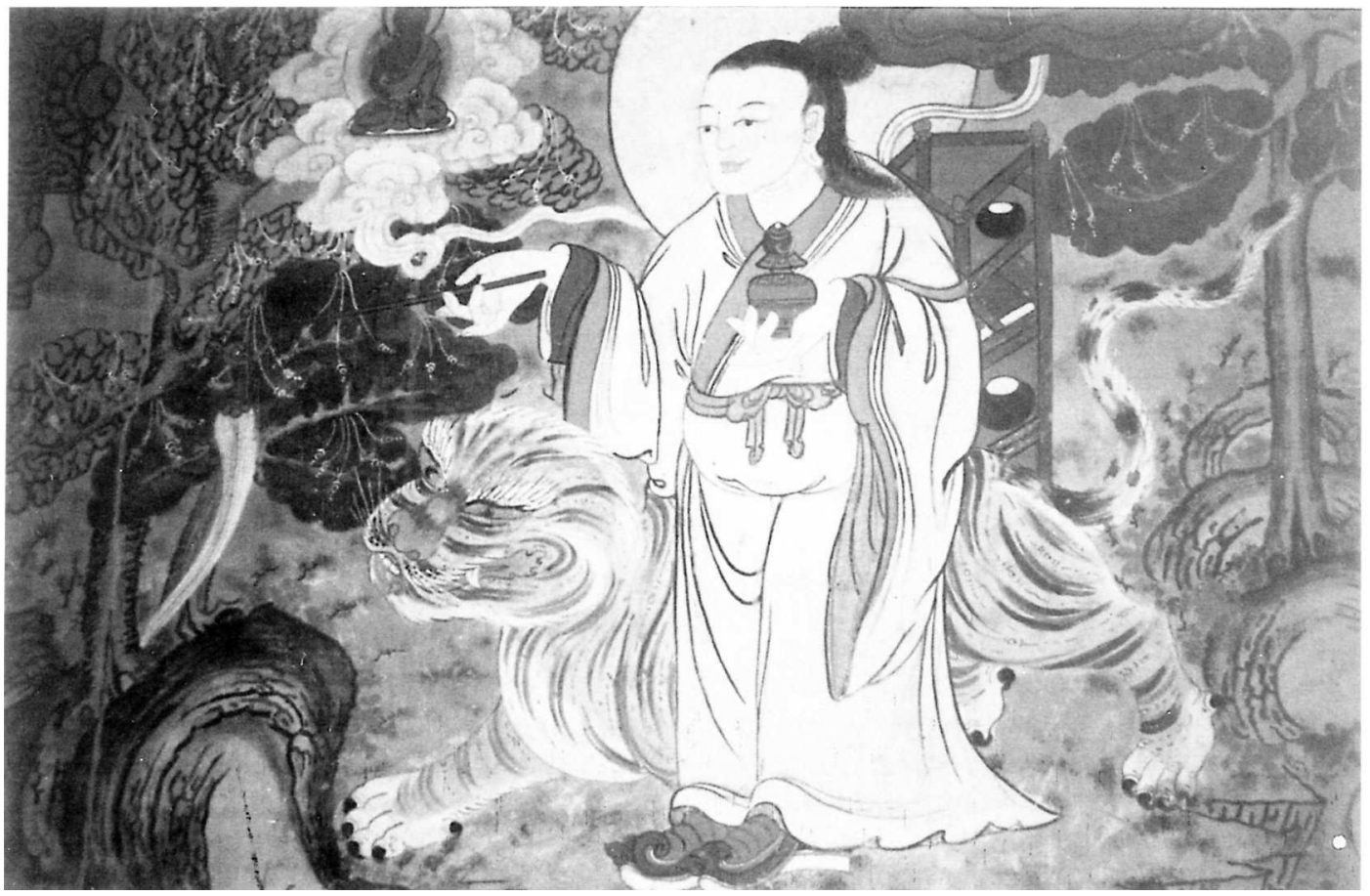


L'homme barbu vêtu comme un moine serait, suivant cette logique, le Mayidari qutuүtu (1593-1635). Nous savons peu de chose de ce grand dignitaire, reconnu comme une incarnation de Maitreya et envoyé à Kökeqota en 1604 à l'âge de douze ans par les autorités religieuses de Lha-sa ${ }^{\text {lxxvi }}$. Sur la peinture, sa barbe, ses longs cheveux et la couleur rouge de sa toge sont caractéristiques des moines des écoles non réformées. Cette obédience est confirmée par une importante source mongole datée de 1662, l'Erdeni-yin tobci, qui présente le Mayidari qutuytu comme la réincarnation de bSam-ba rgya-mtsho, lui-même grand disciple du maître Padmasambhava ${ }^{\text {lxxvii }}$. Or nous savons aujourd'hui que si la réintroduction du bouddhisme en Mongolie est attribuée par les sources tibéto-mongoles de nature apologétique à la seule école réformée des dGe-lugs-pa ${ }^{\text {lxxviii }}$, les écoles non réformées, présentes en Chine et au Tibet oriental, ont également joué un rôle important dans cette renaissance et restent présentes dans toute la Mongolie jusqu'au deuxième quart du XVII ${ }^{e}$ siècle. Le Mayidari-yin juu est bien d'obédience dge-lugs-pa, comme le montre l'ensemble de son décor mural (en particulier les épisodes de la vie de Tsong-kha-pa) ainsi que les autres moines représentés sur la peinture des donateurs, mais a pu être consacré par un moine rnying-ma-pa, comme semblent le rappeler certaines peintures de divinités protectrices qui appartiennent à des cycles rnying-ma-pa ${ }^{\text {lxxix }}$. Le prestige du Mayidari qutuytu éclipse certainement les contradictions provoquées par son obédience, et sa présence semble indiquer que trente ans après la rencontre solennelle en 1578 entre Altan qan et bSod-nams rgya-mtsho, pontife tibétain des dGe-lugs-pa, qui reçoit du chef mongol le titre de Dalai lama, la cohabitation entre les moines « rouges » et les moines «jaunes » ne semble pas poser de problème majeur chez les Tümed.

Jin Shen ${ }^{(4)}$ propose l'identification suivante pour les autres personnages, dont la taille réduite témoigne de l'infériorité de leur rang : la femme (veuve ?) à droite du lama en rouge serait une parente de Macayqatun ; les deux hommes à la gauche de Maca q qatun seraient son jeune petit-fils Möngke tayiji [fig. 11], et à gauche, Sodnam

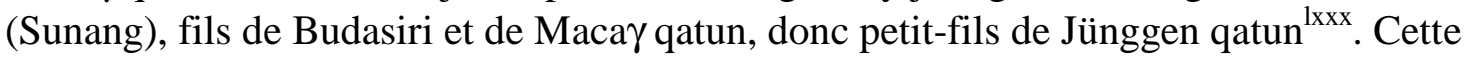
dernière identification repose sur l'air arrogant du personnage, et sur la présence, à l'arrière-plan, d'un soldat tenant des flèches et une sacoche, peut-être symboles de la force militaire de Sodnam. Si la femme au centre du panneau de gauche était Jünggen qatun jeune, l'on ne saurait identifier les deux hommes de gauche : Altan qan avait neuf enfants, dont trois de Jünggen qatun, mais ils ne sont ici que deux. Aussi rejetons-nous cette possibilité.

Les divinités représentées sont deux dieux protecteurs guerriers qui jouent un rôle important dans le bouddhisme mongol. Beg-tse (1Cam-sring, mong. Jamsran), dieu martial protecteur des chevaux, est, selon la légende, soumis et converti au bouddhisme par bSod-nams rgya-mtsho en route pour rencontrer Altan qan sur les berges du Koukounor (Kökenayur). Le Dalai lama le nomme alors protecteur de la foi mongole. Pishamen $^{(7) / V a i c ̧ r a v a n a, ~ p l u s i e u r s ~ f o i s ~ r e p r e ́ s e n t e ́ ~ d a n s ~ l e ~ t e m p l e ~}{ }^{\text {lxxxi }}$, jouit au Mayidari-yin juu d'un culte particulier, qui pourrait remonter à l'idéologie royale d'Altan qan. Le dieu gardien du Nord, région recelant de fabuleux trésors, est non seulement gardien et dispensateur de ses richesses [fig. 10], mais également le protecteur de l'Etat par excellence. 
Fig. 13. Fragment de dessin colorié représentant Abadai qan et son épouse entourés de leur cour. Musée des Beaux-arts d'Oulan-Bator (in Tsultem, 1986, fig. 150.)

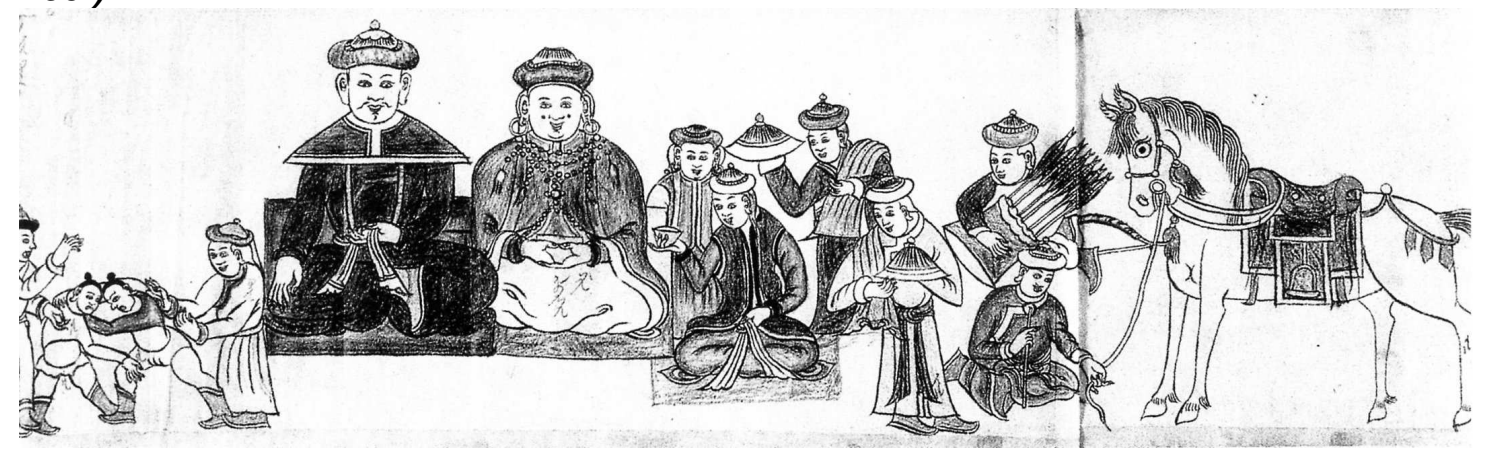

Fig. 14. Donateurs, détail de la partie inférieure d'un thang-ka représentant Vajradhârâ, Tibet oriental (fin XVIIe siècle). Collection privée Robert Hatfield Ellsworth (in Marylin M. Rhie \& Robert A. F. Thurman, 1991, p. 360, cat. 148.)

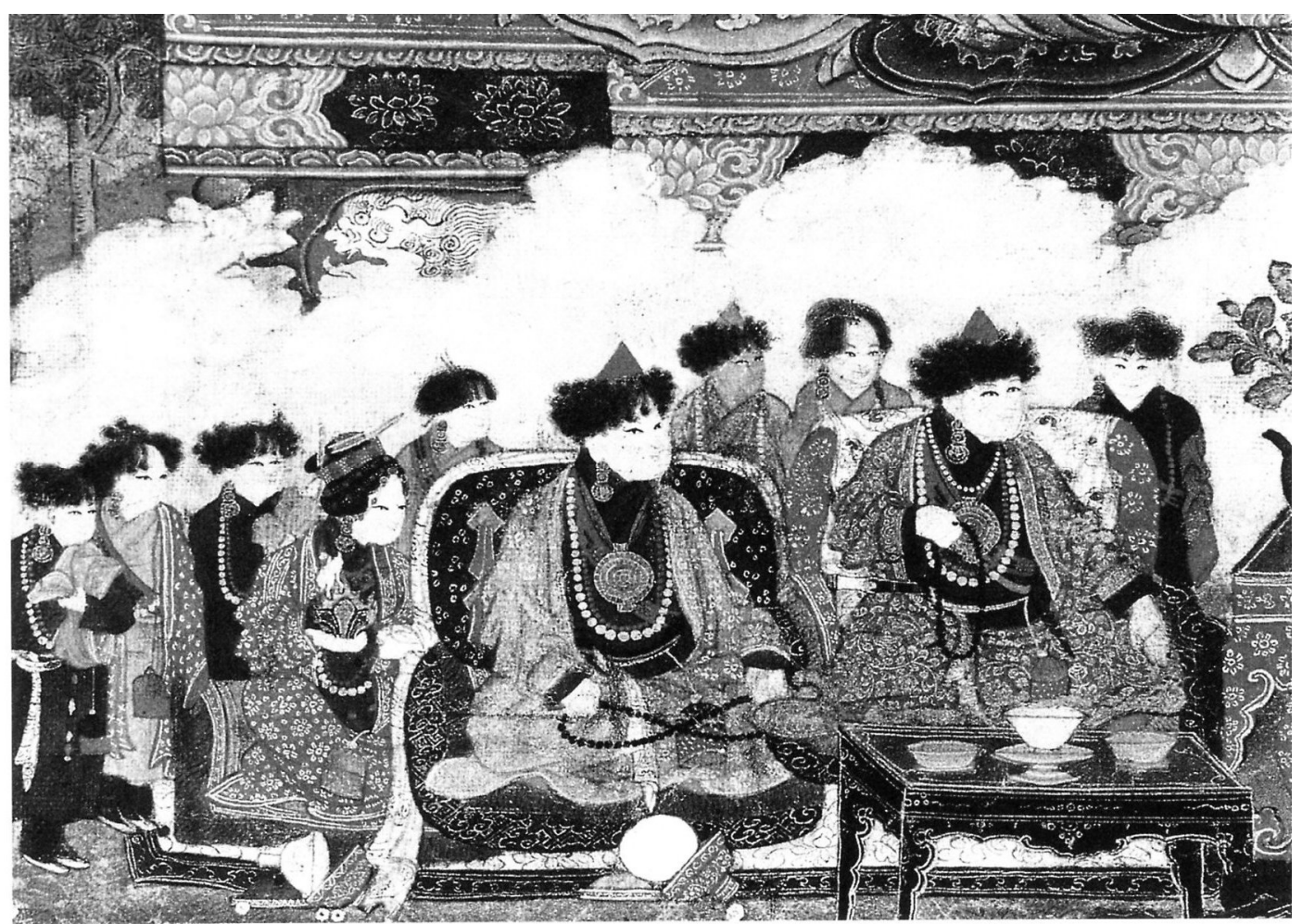

Les deux femmes qui jouent un rôle essentiel dans la politique des Tümed après la mort d'Altan qan occupent le centre des deux panneaux, et cette interprétation correspond au contexte politique et religieux de 1606, date de restauration, de construction dans le temple et de consécration d'une statue. La peinture peut être appelée «peinture des donateurs » car les personnages sont représentés en attitude de prière (rosaire) et d'offrande (cintâmani). Nous sommes également tentés de l'appeler 
peinture d'adoration de Jünggen qatun, considérée comme l'incarnation d'un bodhisattva, par son entourage. Pourrait-il s'agir d'une peinture funéraire, comme le supposent Cheng Xuguang ${ }^{(5)}$ et Liu Yibin ${ }^{(6)}{ }^{1 \times x x i i}$ ? La destination en partie funéraire du Mayidari-yin juu est certes marquée par la présence du stûpa de «l'Impératrice », et par les tombes des descendants d'Altan qan que l'on pouvait voir jusqu'à la révolution culturelle au nord du temple, dans la montagne ${ }^{\text {lxxiii }}$. La composition, et plus particulièrement les musiciens, chanteurs et serviteurs en position d'offrande qui entourent les personnages principaux rappellent les peintures murales des chambres funéraires d'époque Liao et Yuan. Cürüke meurt en 1607, Jünggen qatun, en 1612, tandis que d'autres personnages représentés se soumettent aux Mandchous en 1634. Or compte tenu du déclin rapide du temple après la reddition des Tümed aux Mandchous, la peinture n'est probablement pas postérieure à 1636. En conclusion, la peinture n'a certainement pas été réalisée à l'occasion du décès d'une des deux princesses, et 1606, date de consécration, reste la date la plus probable d'exécution.

La peinture des donateurs est donc un document exceptionnel à plusieurs égards. Sa valeur pour l'histoire des Mongols tümed est unique, même s'il s'agit d'une source difficile à interpréter et retouchée sous les Qing. La richesse et la variété des costumes, des chapeaux et des coiffures font de cette peinture un document ethnographique unique en Mongolie méridionale. Le costume, proche de celui des Yuan, n'indique pas de nette différence de rang social ni de sexe, les serviteurs étant habillés comme les personnages principaux. De plus, le fait que l'on ne décèle aucune influence chinoise importante dans l'apparence extérieure des personnages permet d'infirmer en partie la thèse de l'acculturation des Tümed au début du XVII ${ }^{e}$ siècle $^{\text {lxxxiv }}$.

La valeur artistique de la peinture des donateurs est également exceptionnelle, par le thème représenté comme par le style pictural. Replacée dans le contexte plus large du programme iconographique, qui place donateurs et dharmapâla au-dessous de la vie des saints, elle respecte la disposition traditionnelle de la peinture tibéto-mongole, qu'elle soit mobile ou murale ${ }^{\mathrm{lxxx}}$. La présence des donateurs sur le mur ouest en particulier plutôt que sur les trois autres ne peut cependant parfaitement s'expliquer. Parmi les précédents tibéto-mongols de cette disposition, on peut citer un kesi (tapisserie en soie) du XIV e siècle, tissé dans les ateliers impériaux et montrant, dans le coin inférieur gauche, les empereurs Yuan Qosila (Mingzong, r. 1329) et son frère Tug Temür (Wenzong, r. 1329-32), en attitude de prière, ainsi que leurs épouses leur faisant face, dans le coin inférieur droit. Les empereurs sont placés au même niveau que des dharmapâla, tandis que douze lamas tibétains, peut-être les précepteurs impériaux selon Heather Stoddard, sont placés en haut du kesi ${ }^{\text {ixxvi }}$.

On connaît de remarquables scènes de donateurs royaux au Tibet occidental (temples et grottes de Gu-ge ${ }^{\text {lxxxvii }}$, «scène de libation royale » du 'Du-khang $\mathrm{d}^{\prime} \mathrm{A}-\mathrm{lci}^{{ }^{\mathrm{lxx} x v i i i}}$ ) : comme le Mayidari-yin juu, ce sont des temples royaux centrés sur les statues de culte. Au Tibet central et oriental, les peintures de donateurs laïcs se font plus rares avec la montée des grands pouvoirs religieux. Citons cependant un remarquable thang-ka provenant du Tibet oriental (fin XVII ${ }^{\text {e }}$ siècle), représentant, au-dessous de la divinité principale, des donateurs richement habillés devant des tables d'offrandes (fig. 14). Lorsqu'il est représenté face à un lama pour symboliser la relation mchod-yon $^{\text {lxxxix }}$, qui réunit dans un intérêt commun le lama et le donateur, et dont l'origine remonte à l'histoire du bouddhisme en Inde, le patron laïc est généralement en position 
d'infériorité. Or la peinture des donateurs au Mayidari-yin juu occupe par ses dimensions une place importante dans le décor mural du temple, et les lamas n'y jouent qu'un second rôle puisque les donatrices sont elles-mêmes des incarnations de bodhisattva. Il est donc permis de parler d'une originalité mongole dans le thème représenté.

Fig. 15. Vasudhârâ, registre inférieur du mur intérieur sud de l'entrée du sanctuaire (C) Jin Shen)

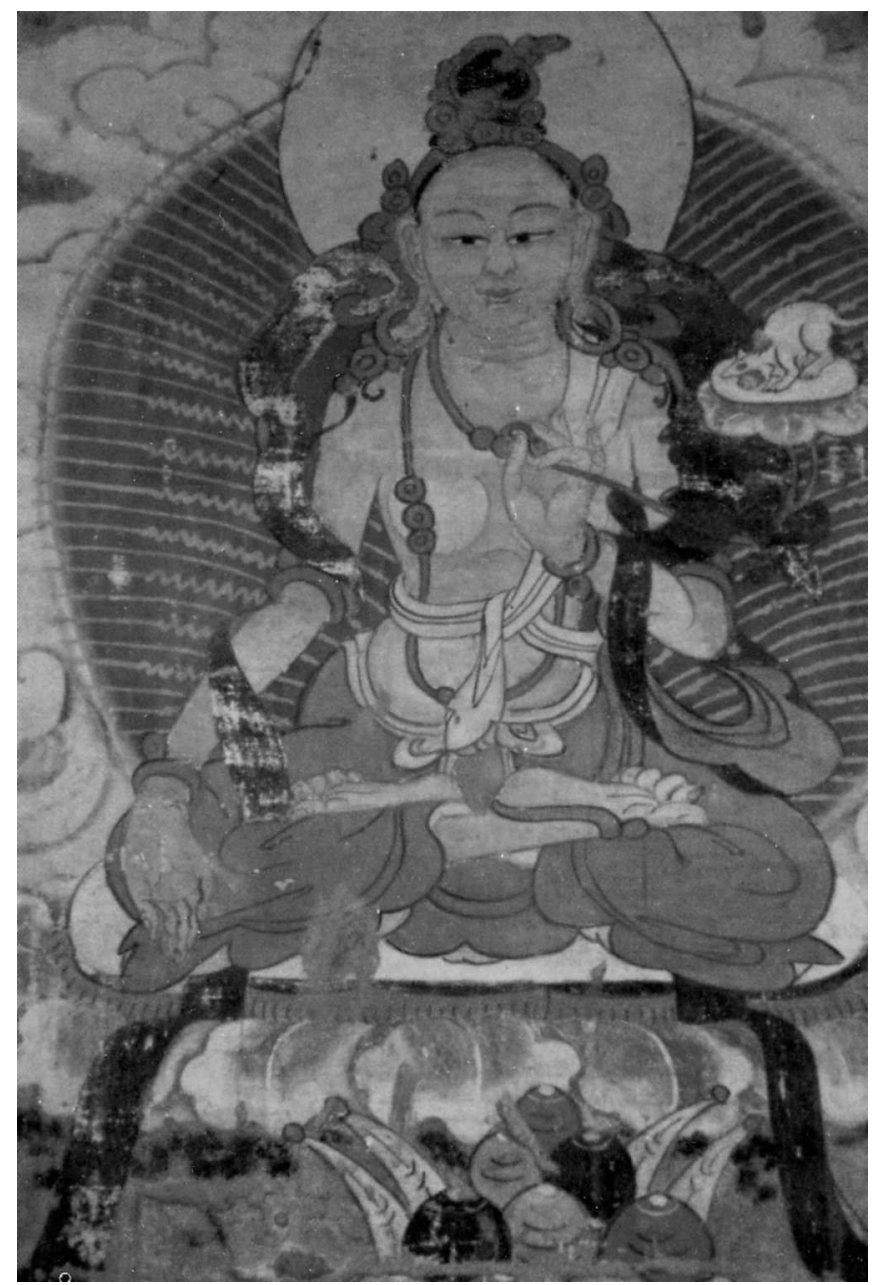

La peinture des donateurs, où tous les personnages, à l'exception de Jünggen qatun et de Pishamen ${ }^{(7)}$, sont vus de trois quart et en pleine action, témoigne de la liberté dont jouissait l'artiste par rapport à la stricte composition des registres religieux. Bien que l'on ne puisse parler de portrait, les visages étant assez stéréotypés ${ }^{\mathrm{xc}}$, les figures, marquées par l'âge, ne sont pas dépourvues d'expression, et une relation émotionnelle semble unir les personnages. D'un point de vue stylistique, la peinture des donateurs peut être comparées à d' autres peintures que l'on peut dater de la période 1580-1632, qui décorent encore aujourd'hui sept édifices tümed ${ }^{\mathrm{xci}}$ ainsi que des grottes récemment découvertes à Arjai (ch. «Baiyanyao ${ }^{(23)}$ ») dans les Ordos ${ }^{\text {xii }}$. Les attitudes, les gestes et le traitement des visages se retrouvent dans les représentations royales de peintures 
mongoles plus tardives (fig. 13) ainsi que dans les donateurs des peintures murales des Yuan et des Ming des grottes de «Baiyanyao ${ }^{(23)}{ }^{\text {xciii }}$. L'ensemble des peintures du temple de Maitreya est rendu très vivant par l'importance des scènes anecdotiques d'un grand naturalisme, que l'on retrouve par exemple dans la représentation de la vie quotidienne (élevages, troupeaux) au Caqar blama juu (fondé en 1583 ou en 1606) ${ }^{\mathrm{xciv}}$. L'influence chinoise est limitée à la représentation des paysages (montagnes, rochers et torrent), à l'iconographie des divinités d'origine chinoise (Pishamen ${ }^{(7)}$ au temple de Maitreya) et à des éléments de détail (corail, joyaux). Le meuble sur lequel est assis Jünggen qatun, la présentation de joyaux ronds, de corail et d'offrandes diverses dans de grands plats posés sur des tables basses font partie du répertoire chinois intégré à la peinture tibétaine. Les détails de joaillerie sont simplifiés, mais le peintre sait rendre les différentes matières (tissus, fourrure, barbe). Les riches boucles d'oreilles sont le seul élément commun aux laïcs et aux bodhisattva représenté sur les autres murs (fig. 10,11) : leur origine est manifestement à rechercher dans la peinture tibétaine. L'arhat Kâlika figuré en peinture dans un des principaux monastères de Kökeqota, le Siregetü juu (temple occidental), tient dans ses mains des boucles d'oreilles similaires ${ }^{\mathrm{xcv}}$.

Deux siècles plus tard, le style du décor mural du Yeke juu (Da zhao $\left.{ }^{(24)}\right)$ de Kökeqota ${ }^{\mathrm{xcvi}}$ adopte des conventions chinoises dans la représentation humaine, mais conserve un certain nombre des caractéristiques de ces premières peintures murales (fond de verdure, goût du détail anecdotique, silhouette et parure des bodhisattva etc.). Ces décors muraux ayant fait l'objet de très peu de publications, il est encore trop tôt pour affirmer l'existence d'un corpus pictural tümed et ordos homogène et d'une filiation stylistique avec la peinture Yuan, et il faudra attendre une étude approfondie ainsi que la publication détaillée de ces peintures.

\section{BIBLIOGRAPHIE}

Aletan han zhuan ${ }^{(42)}$ [«Biographie d'Altan qan»], Auteur anonyme, inspiré de l'oeuvre d'Uran Tangyari Dayun Kiya, ère Wanli (1572-1619), peut-être 1610-1614, trad. du mongol

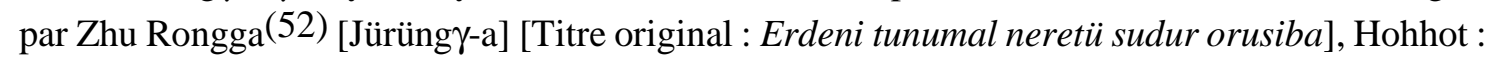
Nei Menggu renmin chubanshe, 1991.

Bawden, Charles R., 1955, The Mongol Chronicle of Altan Tobci, texte, trad. et notes critiques, Wiesbaden : Otto Harrassowitz, 1955 (Göttinger Asiatische Forschungen, 5.)

Berger, Patricia et al., 1995, Mongolia: the Legacy of Chinggis Khan, Londres \& New-York : Thames and Hudson; San Francisco (Calif.) : Asian Art museum of San Francisco.

Boyer, Martha, 1952, Mongol Jewelry, Copenhague : Gyldendalske Boghandel, Nordisk Forlag.

Cammann, Schuyler, 1963, « Mongol Costume - historical and recent », p. 156-166 in Aspects of Altaic Civilization, Proceedings of the Fifth meeting of the PIAC (1962), Denis Sinor éd., Bloomington : Indiana University Press, 1963, (Uralic and Altaic Series, 23).

Charleux, Isabelle, décembre 1998, Histoire et architecture des temples et monastères lamaïques de Mongolie méridionale (thèse de Doctorat, Université de Paris-Sorbonne), Paris. 
Charleux, Isabelle, «La peinture des donateurs du temple de Maitreya en Mongolie méridionale » Author's own file, not the published version

Please see the published version in Arts Asiatiques 54 (1999), p. 85-102

Chavannes, Edouard, janv-fév. 1896, «A. Pozdnéieff, Sur un monument nouvellement découvert de la littérature mongole au temps de la dynastie Ming », Journal Asiatique 7 (Paris), p. 173-179.

Cheng Xuguang(5) \& Liu Yibin(6), mai 1984, «Meidai zhao zhaomiao jianzhu, bihua yishu kaocha baogao $(53)$ [«Rapport d'enquête sur l'architecture et la peinture murale du Mayidari-yin juu »], Baotou shiliao huiyao (54)10, p. 178-183.

Even, Marie-Dominique \& Pop, Rodica, trad. et ann., 1994, Histoire secrète des Mongols (Mongghol-un ni'uca tobciyan) : Chronique mongole du XIIIème siècle, Paris : Gallimard (Connaissance de l'Orient.)

Goepper Roger, 1996, « Murals in the Early Temples of Alchi », p. 226-243 in On the Path to Void, Buddhist Art of the Tibetan Realm, Pratapaditya Pal éd., Mumbai (Inde) : Marg.

Hansen, Henning Harald, 1950, Mongol Costumes, Researches on the garments collected by the first and second Danish Central Asian Expeditions under the Leadership of Henning Haslund-Christensen, Copenhague.

Hansen, Henning Harald \& Nicolaisen Ida éd., 1993, Mongol Costumes, Copenhague \& Londres : The Carlsberg Fundation's Nomad Research Project, Thames and Hudson / Copenhague : Rhodos International Science and Art Publishers.

Heissig, Walther, 1954, Die Pekinger Lamaistischen Blockdrucke in mongolischer Sprache, Wiesbaden : Otto Harrassowitz (Göttinger Asiatische Forschungen, 2).

Heissig, Walther \& Dumas, Dominique, 1995, The Mongols [Die Mongolen], Innsbruck : Pinguin; Francfort : Umschau.

Jin Shen(4), 1984a, Meidai zhao(25) [«Le Mayidari-yin juu »], Hohhot : Nei Menggu renmin chubanshe.

Jin Shen ${ }^{(4)}$, 1984b, « Meidai zhao bihua »(55) [« Peintures murales du Mayidari-yin juu »], Nei Menggu shehui kexue (56) 1984-2 (Hohhot), p. 116-120.

Jin Shen(4), mai 1984c, « Meidai zhao bihua chutan(57)» [«Recherches préliminaires sur les peintures murales du Mayidari-yin juu »], Baotou shiliao huiyao (54) 10 (Hohhot), p. 166-177.

Jin Shen(4), s. d. (v. 1980), «Meidai zhao ji qi bihua(58)» [«Le Mayidari-yin juu et ses peintures murales »], Baotou wenwu ziliao(59) 1 (Hohhot), p. 174-184.

Jin Weinuo(45) éd., 1991, Zhongguo bihua quanji, 32 : Zangchuan siyuan, 2(60) [« Collection complète des peintures murales de Chine, 32 : les monastères tibétains, $2 »]$, Tianjin : Tianjin renmin meishu chubanshe (Zhongguo meishu fenlei quanji.)

Kessler, Adam T. et al., 1993, Empires beyond the Great Wall, The Heritage of Genghis Khan, Los Angeles : Natural History Museum of Los Angeles County. 
Charleux, Isabelle, «La peinture des donateurs du temple de Maitreya en Mongolie méridionale » Author's own file, not the published version

Please see the published version in Arts Asiatiques 54 (1999), p. 85-102

Kler, Joseph (CICM), 1957, «Travaux d'orfèvrerie aux pays des Ordos », Central Asiatic Journal, 2(4) (Wiesbaden : Mouton ; La Haye : Otto Harrassowitz), p. 188-203.

Kurakichi Shiratori, 1929, « The Queue among the People of North Asia », The Memoirs of the Research Department of the Toyo Bunko 4 (Tôkyô), p. 1-69.

Lalou, Marcelle, 1930, Iconographie des étoffes peintes (pata) dans le Mañjuçrîmûlakalpa, Paris : Paul Geuthner.

Lessing, Ferdinand D., 1995 [1960], Mongolian-English Dictionary, Bloomington (Indiana) : The Mongolia Society [Berkeley; Los Angeles : University of California Press].

Li Yiyou(40), mai 1980, « Tumo chuan shang de di yi zuo mingdai chengsi : Meidai zhao(61)» [«Le Mayidari-yin juu, première ville-temple d'époque Ming à Tumochuan »], Baotou shiliao huiyao(54) 1, p. 183-192.

Mostaert, Antoine (CICM), 1927, «A propos de quelques portraits d'empereurs mongols », Asia Major 4 (Leipzig), p. 147-156.

Mostaert, Antoine, 1956, « Matériaux ethnographiques relatifs aux Mongols Ordos », Central Asiatic Journal, 2(4) (Wiesbaden : Mouton ; La Haye : Otto Harrassowitz), p. 241-294.

Pozdneev, Aleksei Matveevich, 1895, «Novo-otkrity pamyatnik mongolskoy pismenosty vremën dinastii Ming », Vostochniye Zametky 14 (Saint-Pétersbourg), p. 367-381.

Qing Chunsong(33), 1983/4, «Nei Menggu Chifeng shi Yuan Baoshan Yuan mu bihua (62) [«Peintures murales d'une tombe d'époque Yuan à Yuanbao shan(32), Chifeng shi, Mongolie-Intérieure »], Wenwu(63), p. 40-46.

Rhie, Marylin M. \& Thurman, Robert A. F., 1991, Wisdom and Compassion : The Sacred Art of Tibet, New York : Asian Art Museum of San Francisco and Tibet House.

Rong Xiang(41), 1979, Huhehaote yange jiyao gao(64) [«Esquisse de l'évolution historique de Hohhot »], Hohhot : Nei Menggu shehui kexueyuan.

Roudova, Maria, 1988, Chine, Coutumes et traditions dans l'imagerie populaire, trad. du russe par Marianne Dunlop, Léningrad : Ars Mundi ; Aurora.

Schmidt, Isaac Jacob, 1961 [1829], Geschichte der Ost-Mongolen und ihres Fürstenhauses, verfasst von Ssanang Ssetsen Chungtaidschi der Ordus ; aus dem Mongolischen übersetzt, und mit dem Originaltexte...herausgegeben, trad. de l'Erdeni-yin tobci [ «Chroniques de joyau »] de Sayang Secen, 1662, La Haye : Europe Printing [Saint-Pétersbourg].

Serruys, Henry (CICM), 1945, «Le Pei-lou fong-sou. Les coutumes des esclaves septentrionaux », trad. et notes critiques du Beilu fengsu(12) (dit aussi Yisu ji(65)) de Xiao Daheng(31), Monumenta Serica 10 (Pékin \& Tôkyô), p. 117-164 (rééd. inThe Mongols and Ming China : Customs and History, 1987, I). 
Charleux, Isabelle, «La peinture des donateurs du temple de Maitreya en Mongolie méridionale » Author's own file, not the published version

Please see the published version in Arts Asiatiques 54 (1999), p. 85-102

Serruys, Henry, 1957, « Remains of Mongol Customs in China during the early Ming Period », Monumenta Serica 16 (Pékin \& Tôkyô), p. 137-190 (rééd. inThe Mongols and Ming China : Customs and History, II).

Serruys, Henry, avril-juin 1958, « Notes on a Chinese Inscription of 1606 in a lamaist Temple in Mai-ta-chao Sui-yüan », Journal of the American Oriental Society 78(2), p. 101-113.

Serruys, Henry, 1975a, « Jünggen, a title of Mongol Princess », Ural-Altaische Jahrbücher 47 (Wiesbaden), p. 177-185.

Serruys, Henry, 1975b, «Two remarkable Women in Mongolia : The Third Lady Erketï Qatun and Dayicing beyiji », Asia Major 19 (Londres), p. 191-245 (rééd. inThe Mongols and Ming China : Customs and History, VIII).

Serruys, Henry, 1987, The Mongols and Ming China : Customs and History, édité par Françoise Aubin, Londres : Variorum Reprints.

Singer, Jane Casey, 1995, «Early portrait painting in Tibet », p. 81-99 in Function and Meaning in Buddhist Art, Proceedings of a seminar held at Leiden University, 21-24 oct. 1991, K. R. van Kooij \& H. van der Veer éd., Groningen : Egbert Forsten.

Steinhardt, Nancy Shatzman, 1990/1, «Yuan Period Tombs and their Decoration : Cases at Chifeng », Orientations 36(4) (Hong-Kong), p. 198-221.

Su Bai(28), 1994/4, « Huhehaote ji qi fujin ji zuo zhaomiao diantang buju de chubu tantao »(66) [«Examen du plan de quelques bâtiments monastiques de Hohhot et des environs »], Wenwu(63) (Pékin), p. 53-61.

Tsultem, N., 1986, Bref aperçu sur le développement de la peinture mongole «Mongol zourag », Oulan-Bator : Section de la Publication d'Etat.

Wada Sei (= Wada Kiyoshi(44)) (=ch. Hetian Qing), 1984 [1959], Mingdai Menggu shi lunji(67) [«Recueil d'essais sur l'histoire de la Mongolie sous les Ming »], trad. en chinois par Pan Shixian(68), Pékin : Shangwu yinshu guan (titre original : Tôashi kenkyû : Môko hen(69) [«Etude de l'histoire de l'Extrême-Orient : la Mongolie »], Tôkyô, 1959.)

Wanli yehuo bian(21) [ «Collection d'anecdotes réunies informellement sous l'ère Wanli »], de Shen Defu(22) (1578-1642), 1606, et supplément 1619, ed. Pékin : Zhonghua shuju, 1997 [1959].

Wang Bomin(30) éd., 1995, Zhongguo shaoshu minzu meishu shi(70) [« Histoire de l'art des minorités de Chine »], Fuzhou : Fujian chubanshe.

Wang Dafang(49), Batu Jirigala(50) (Batu Jirgala) \& Zhang Wenfang(51), 1994, « Baiyanyao shiku de yingjian niandai ji bihua zhuyao neirong chulun »[71] [«Première discussion du contenu des peintures murales et de la période d'installation dans le site rupestre de Baiyanyao(23) »], p. 566-578 in Nei Menggu wenwu kaogu wenji (72), Li Yiyou(40) éd., vol. 1, Pékin : Zhongguo da baike quanshu chubanshe. 
Charleux, Isabelle, «La peinture des donateurs du temple de Maitreya en Mongolie méridionale » Author's own file, not the published version

Please see the published version in Arts Asiatiques 54 (1999), p. 85-102

Wang Degong(27), s. d. (v. 1980), «Meidai zhao Taihe men shike tiji zhengwu»(73) [«Rectification concernant l'inscription en pierre de la porte Taihe au Mayidari-yin juu »], Baotou wenwu ziliao(59) 1, (Hohhot), p. 185-186.

\section{Glossaire chinois et japonais}

(1) 呼和浩特

(2) 大青山

(3) 三娘子

(4) 金申

(5) 程旭光

(6) 劉毅涁

(7) 毗沙門

(8) 多耳天王

(9) 雲旗

(10) 二胡

(11) 古琴

(12) 北虜風俗

(13) 太后廟

(14) 蒂帽

(15) 渾脫帽

(16) 暖帽

(17) 姑姑

(18) 質孫服

(19) 比甲

(20) 福

(21) 萬曆野獲編

(22) 沈德符

(23) 百眼䇺

(24) 大召

(25) 美岱召

（26）明實錄

(27) 王德恭

(28) 宿白

(29) 金峰

(30) 王伯敏

(31) 蕭大亨

(32) 元寶山

(33) 頃春松

(34) 元朝帝后畫像

(35)賈哈

(36) 元史

(37) 后妃傳

(38) 劉貫道

(39) 永和宮

(40) 李逸友

(41) 榮祥

(42) 阿勒坦汗傳
(43) 財神廟

(44) 和田清

(45) 金維諾

(46) 阿爾些石窟

(47) 阿爾巴斯

(48) 鳥蘭鎭

(49) 王大方

(50) 巴圖吉日嘎拉

(51) 張文芳

(52) 珠榮嘎

（53）美岱召召廟建築、壁畫藝術考察報告

(54) 包頭史料薈要

(55) 美代召壁畫

(56) 内蒙古社會科學

(57) 美代召壁畫初探

(58) 美代召及其壁畫

(59) 包頭文物資料

(60) 中國璧畫全集: 藏傳寺院

(61) 土默川上的第一座明代城寺- - 美岱召

(62) 内蒙古赤峰市元寶山元墓壁畫

(63) 文物

(64) 呼和浩特沿革紀要稿

(65) 夷俗記

(66) 呼和浩特及其附近几座召廟殿堂布局的初步探討

(67) 明代蒙古史論集

(68) 潘世憲

(69) 東亞史研究 : 蒙古篇

(70) 中國少數民族美術史

(71) 百眼窯石窟的營建年代及壁畫主要内容初論

(72) 内蒙古文物考古文集

(73) 美岱召泰和門石刻題記證誤 


\section{RÉSUMÉS}

Le Mayidari-yin juu, temple bouddhique fortifié fondé en 1575 par Altan qan des Tümed à $70 \mathrm{~km}$ à l'ouest de Hohhot en Mongolie méridionale (Région Autonome de Mongolie-Intérieure, Chine), a conservé un important décor mural que l'on peut dater du début du XVII siècle. Les donateurs mongols qui ont financé les constructions et réparations de 1606 sont représentés dans le registre inférieur du mur occidental du sanctuaire. Jünggen qatun, célèbre troisième femme d'Altan qan, et Maca q qatun faisant face au Mayidari qutuytu occupent le centre des deux parties de la peinture. La variété des costumes, des chapeaux et des coiffures fait de cette peinture un document ethnographique unique en Mongolie méridionale. La peinture des donateurs est ainsi le résultat de la rencontre entre religieux de diverses obédiences et artistes mongols, chinois et tibétains.

\section{The painting of donors of Maitreya temple in Southern Mongolia}

The Mayidari-yin juu, a fortified Buddhist temple founded in 1575 by Altan qan of the Tümed tribe, at $70 \mathrm{~km}$ West of Hohhot in Southern Mongolia (Inner Mongolia Autonomous Region, China), preserved important murals painted during the first half of the 17th. Mongol donors who financed the constructions and restaurations in 1606 are portrayed in the lower part of the Western wall of the sanctuary. Jünggen qatun, the

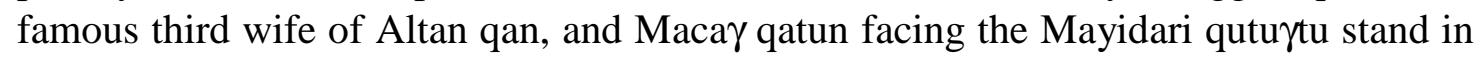
the middle of the two parts of the painting. The variety of costumes, hats and hairstyles makes this painting a unique ethnographical document in Southern Mongolia. This mural is thus the result of the encounter between religious from different schools and Mongolian, Chinese and Tibetan artists.

\footnotetext{
${ }^{\mathrm{i}}$ Nous remercions vivement Marie-Dominique Even et Caroline Gyss-Vermande pour avoir accepté de relire cet article.

${ }^{\text {ii }}$ Huhehaote $^{(1)}$ suivant la transcription pinyin, et Hohhot suivant la transcription chinoise à usage international.

${ }^{\text {iii }}$ Ceux-ci fondent la dynastie Qing en 1636 et conquièrent la Chine en 1644. Les tribus septentrionales (mongols Qalqa) font acte d'allégeance aux Mandchous en 1691.

${ }^{\text {iv }}$ Ch. Meidai zhao ${ }^{(25)}$ dans la transcription chinoise la plus courante.

${ }^{\mathrm{v}}$ Bien que le bouddhisme tibétain soit proclamé religion officielle sous la dynastie Yuan (1277-1368), les Mongols retournent à leurs croyances chamaniques lors de leur repli en Mongolie après la chute de la dynastie.

${ }^{\text {vi }}$ C'est ce que semblent suggérer les Annales dynastiques de la dynastie Ming (Ming shilu ${ }^{(26)}$ ) ainsi que d'autres sources contemporaines. Charleux, 1998, p. 34-37, 44-46. Le temple se trouve aujourd'hui au milieu des champs, à proximité de l'autoroute Hohhot-Baotou. Il est géré comme un musée par l'Administration du patrimoine de la ville de Baotou, et aisément visitable ; nous nous sommes rendue sur place en 1993 et en 1998.

${ }^{\text {vii }}$ Le lévirat était pratique courante chez les Mongols : la veuve demeurait dans la famille du mari défunt et était donnée en mariage à un cadet ou à un fils de ce dernier. Jünggen qatun épouse successivement Altan qan, puis le fils aîné, le petit-fils et enfin l'arrière petit-fils d'Altan qan. Dans ce cas, le lévirat était
} 
Charleux, Isabelle, «La peinture des donateurs du temple de Maitreya en Mongolie méridionale »

Author's own file, not the published version

Please see the published version in Arts Asiatiques 54 (1999), p. 85-102

encouragé par la cour des Ming qui voyait dans les remariages de Jünggen qatun une garantie de paix. Serruys, 1975b, p. 191-245 ; Serruys, 1975a, p. 177-185.

viii Pour la traduction et l'interprétation de cette inscription, voir Charleux, 1998, p. 68-71 ; Serruys, 1958, p. 101-113 et Wang Degong ${ }^{(27)}$, s. d. (v. 1980), p. 185-186.

${ }^{\text {ix }}$ L'architecture du Mayidari-yin juu n'a aucun équivalent en Mongolie. La date des bâtiments, leur libre organisation à l'intérieur de l'enceinte, et surtout leurs orientations différentes sont l'objet de controverses (par exemple : Su Bai ${ }^{28}$, 1994/4, p. 55-56). Pour une description et une étude de ce temple, voir Charleux, 1998, p. 316-344.

${ }^{\mathrm{x}}$ Il s'agit du Bouddha Çâkyamuni entouré de scènes narratives sur le mur nord, et de Tsong-kha-pa entouré des épisodes de sa vie sur les murs est et ouest. Les peintures du mur sud, de part et d'autre de l'unique porte, représentent des guerriers et divinités à têtes animales évoquant celles qui apparaissent au défunt durant le bar-do («état intermédiaire » entre la mort d'un être et sa renaissance suivante).

${ }^{\mathrm{xi}}$ Jin Shen ${ }^{(4)}$, 1984a ; Jin Shen ${ }^{(4)}$, 1984b ; Jin Shen ${ }^{(4)}$, 1984c ; Jin Shen ${ }^{(4)}$, s. d. ; Jin Shen ${ }^{(4)}$, 1984c ; Cheng Xuguang $^{(5)} \&$ Liu Yibin ${ }^{(6)}, 1984$.

xii Jin Shen ${ }^{(4)}$, 1984c, p. 175 ; Jin Shen(4), s. d., p. 182 ; Cheng Xuguang ${ }^{(5)} \&$ Liu Yibin ${ }^{(6)}, 1984$, p. 180. Cheng Xuguang(5) et Liu Yibin( ${ }^{(6)}(1984$, p. 178-180) pensent que la peinture de donateurs doit être datée de l'époque Qing et recouvre un programme différent, mais ces auteurs ne mentionnent pas les constructions de 1606. Jin Feng ${ }^{(2)}$, interrogé à ce propos en 1990 et 1991, tente de démontrer que la peinture n'a subi aucune modification depuis les Ming (Wang Bomin ${ }^{(30)}$ éd., 1995, vol. 1, p. 185, n. 11).

xiii Il s'agit d'une peinture a tempera utilisant une dizaine de pigments minéraux et des oxydes métalliques délayés dans de l'eau additionnée d'un agglutinant. Ces pigments sont appliqués sur un enduit qui recouvre un crépi dont la surface, une fois séchée, a été soigneusement lissée.

${ }^{x i v}$ Hansen \& Nicolaisen éd., 1993 ; Heissig \& Dumas, 1995.

${ }^{\mathrm{xv}}$ Pour une description du costume mongol au début du XX $\mathrm{X}^{\mathrm{c}}$ siècle, voir Mostaert, 1956, p. 265-267 ; Hansen \& Nicolaisen éd., 1993.

${ }^{\text {xvi }}$ Beilu fengsu ${ }^{(12)}$, de Xiao Daheng ${ }^{(3)}$ (1532-1612), traduit et annoté par Serruys, 1945.

${ }^{x v i i}$ Beilu fengs $u^{(12)}$, trad. Serruys, 1945, p. 138.

${ }^{x v i i i}$ Beilu fengsu ${ }^{(12)}$, trad. Serruys, 1945, p. 144.

${ }^{\text {xix }}$ Sous les Yuan, les Mongols avaient, de même que les Jürced et les Kitan, coutume de se raser les parties latérales de la tête et le haut du front, en laissant deux nattes derrière le crâne ou des mèches derrière les oreilles, comme le montrent les peintures murales Yuan des tombes de Yuanbao shan ${ }^{(32)}$ près de Chifeng (Steinhardt, 1990/1, p. 198-221 ; Qing Chunsong(33), 1983/4, p. 40-46) ; les «Portraits d'empereurs et d'impératrices de Chine » («Yuanchao dihou huaxiang $\left.{ }^{(34)} »\right)$, conservés au Gugong de Pékin (étudiés par Mostaert, 1927, p. 147-156) ; le portrait de Qubilai attribué à Anige (1294, feuille d'album, couleurs et encre sur soie, musée du Palais, Taipei). Voir également Kurakichi Shiratori, 1929, p. 30 sq. ; Jin Shen ${ }^{(4)}$, 1984b, p. 117 et Jin Shen ${ }^{(4)}$, s. d., p. 180.

${ }^{\mathrm{xx}}$ On coupait la natte aux prisonniers qui perdaient ainsi une part de leur identité.

${ }^{x x i} L^{\prime}$ ' Impératrice » pourrait désigner l'une des deux donatrices, Jünggen qatun ou Maca q qatun.

xxii Jin Shen ${ }^{(4)}$, s. d., p. 180.

${ }^{\text {xxiii }}$ Altan tobci, éd. trad. Bawden, 1955, p. 107 ; Erdeni-yin tobci, éd. et trad. Schmidt, 1961 [1829], p. 210. Le traité de paix renoue les relations politiques et commerciales sino-mongoles et met fin aux razzias tümed en rétablissant les marchés frontaliers. Les Ming achètent en réalité la paix très cher, et sont soulagés de négocier car leurs défenses sont pauvres. Si les Tümed payent un tribut annuel, ils reçoivent de nombreux cadeaux cérémoniels proportionnels à l'importance de ce tribut, ainsi que des cadeaux supplémentaires (or, argent, rouleaux de soie...) et vendent aux Chinois des chevaux à un prix élevé.

${ }^{\text {xxiv }}$ Beilu fengs $u^{(12)}$, trad. Serruys, 1945, p. 144.

${ }^{x x v}$ Jin Shen ${ }^{(4)}$, 1984b, p. 118 ; Jin Shen ${ }^{(4)}$, s. d., p. 181.

${ }^{x x v i}$ Les femmes des Ordos ont les cheveux peignés en « tresses géminées dont les extrémités, enfermées dans des tubes de toile rigide brodée et chargée chez les riches de grosses plaques d'argent travaillées, sont rattachées à la ceinture par des rubans brodés appropriés » (Kler, 1957, p. 198). Les pièces de tissu qui les recouvrent peuvent être attachées à la ceinture. Des coiffures semblables conservées dans les collections danoises et mongoles caractérisent les Ordos, Üjümücin, Dörbed ainsi que les Bouriates -les Tümed ayant 
Charleux, Isabelle, « La peinture des donateurs du temple de Maitreya en Mongolie méridionale » Author's own file, not the published version

Please see the published version in Arts Asiatiques 54 (1999), p. 85-102

probablement adopté d'autres modes sous les Qing (Heissig \& Dumas, 1995, p. 27, 29, 32 , 33 ; Boyer, 1952, p. 123 ; Mostaert, 1957, p. 269). Chez les Bouriates, les jeunes femmes non mariées portent sept tresses puis deux quand elles se marient (Jin Shen ${ }^{(4)}, 1984$ b, p. 118).

xxvii Jin Shen ${ }^{(4)}$, s. d., p. 179 ; Jin Shen ${ }^{(4)}$, 1984c, p. 171 (reproduit fig. 6, p. 171).

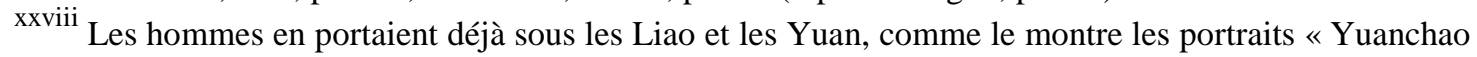
dihou huaxiang ${ }^{(34)} \gg$ du Gugong de Pékin.

${ }^{\text {xxix }}$ Steinhardt, 1990/1, p. 199.

${ }^{\mathrm{xxx}}$ Les femmes ordos portent une coiffure lourde d'argenterie : couronne et portique en miniature, disques d'argent travaillés à jour, et amalgame de chaînettes, torsades et breloques pendant sur chaque joue, turquoise et larges perles de corail ornant les boîtes en argent évidées qui protègent les tresses (Kler, 1957, p. 198 et fig. 8, p. 199 ; Mostaert, 1956, p. 269). Les femmes qalqa, üjümücin ont également une coiffure sophistiquée (Heissig \& Dumas, 1995, p. 21-23, 28...).

${ }^{x x x i}$ Beilu fengsu ${ }^{(12)}$, trad. Serruys, 1945, p. 145.

${ }^{\text {xxii }}$ Par exemple le chapeau d'hiver du huitième Boydo gegen de Da küriye (Berger et al., 1995, cat. 20 p. 140). Voir également : Heissig \& Dumas, 1995, p. 27 ; Tsultem, 1986, fig. 114, 150, 160...

xxxiii Jin Shen ${ }^{(4)}$, s. d., p. 181 ; Jin $\operatorname{Shen}^{(4)}$, 1984b, p. 118.

${ }^{\text {xxxiv }}$ Des Mongols le portent sur des peintures de Dunhuang : «Procession de Mongols », grotte $n^{\circ} 332$ de Mogao (époque Yuan), reproduit dans Steinhardt, 1990/1, fig. 24, p. 212.

${ }^{\mathrm{xxv}}$ Beilu fengsu ${ }^{(12)}$, trad. Serruys, 1945, p. 145.

${ }^{x x x v i}$ Voir les peintures Xixia de Dunhuang, grotte 418. Jin Shen ${ }^{(4)}$, 1984b, p. 118-119.

xxxvii Jin Shen ${ }^{(4)}$, 1984b, p. 118.

${ }^{\text {xxxviii }}$ Peintures de Yuanbao shan ${ }^{(32)}$ (Steinhardt, 1990/1, fig. 2, p. 199) ; portraits de Gengis Khan et de Qubilai (musée du Palais de Taipei). Jin Shen(4), 1984b, p. 119.

${ }^{\text {xxix }}$ Jin Shen $^{(4)}$, s. d., p. 179 ; Jin Shen ${ }^{(4)}$, 1984c, p. 171 (reproduit fig. 6, p. 171).

${ }^{x l}$ Cammann, 1963, p. 156-166; Mostaert, 1956, p. 268.

${ }^{x l i}$ Beilu fengsu $u^{(12)}$, trad. Serruys, 1945, p. 136 et p. 136, n. 29.

xlii Depuis les Yuan, le pan à boutonnières de leur habit est attaché du côté droit.

xliii Jin Shen ${ }^{(4)}$, s. d., p. 180 ; Jin Shen ${ }^{(4)}, 1984$ b, p. 120.

${ }^{\text {xliv }}$ Voir par exemple les portraits « Yuanchao dihou huaxiang ${ }^{(34)} »$ du Gugong de Pékin, et la peinture de Yuanbao $\operatorname{shan}^{(32)}$. Steinhardt, 1990/1, fig. 2, p. 199. Les cafetans de la peinture correspondent à la description du Beilu fengsu ${ }^{(12)}$ (trad. Serruys, 1945, p. 145).

${ }^{x l v}$ Jin $\operatorname{Shen}^{(4)}$ (s. d., p. 180) nomme cette cape $g u h a^{(35)}$.

${ }^{x l v i}$ On ne voit pas sur la peinture les manches terminées «en sabot de cheval » très fréquentes sous les Qing.

${ }^{\text {xlvii }}$ Voir par exemple les peintures de Yuanbao shan $^{(32)}$. Steinhardt, 1990/1, p. 199 ; également Cammann, 1963, p. 156-166.

xlviii Lessing, 1995 [1960], p. 865.

${ }^{x l i x}$ Yuan shi $^{(36)}$, «Houfei zhuan »(37), cité par Jin Shen ${ }^{(4)}$, s. d., p. 182 ; Jin Shen ${ }^{(4)}, 1984$ b, p. 119. Ce gilet est porté sous les Qing par les femmes caqar : Hansen \& Nicolaisen éd., 1993, cat. 78, 80, 113, 120.

${ }^{1}$ Cammann, 1963, p. 156-166 ; Mostaert, 1956, p. 265. Chez les Mongols qalqa, l'épouse est appelée «celle qui est sans ceinture » (büse-ügei).

${ }^{\mathrm{li}}$ Histoire secrète des Mongols, trad. Even \& Pop, 1994, p. 71. Les vaincus ôtent leur ceinture en signe de soumission.

${ }^{\text {lii }}$ Hansen,1950, emploie le mot toge, Cammann,1963, préfère employer le mot grec chiton.

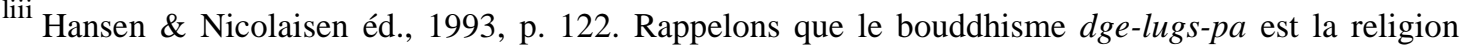
«jaune » (sira-yin shasin) ou d'or (altan nom, «la Loi d'or»), les moines étant appelés «bonnets jaunes » en raison de la couleur de leurs chapeaux.

${ }^{\text {liv }}$ Voir par exemple les peintures de Yuanbao shan ${ }^{(32)}$ (Steinhardt, 1990/1) ; la peinture de Liu Guandao ${ }^{(38)}$ intitulée «Qubilai qan chassant» (encre et couleurs sur soie, 1280, musée du Palais, Taipei) ; les «Yuanchao dihou huaxiang ${ }^{(34)} »$ du Gugong de Pékin. 
Charleux, Isabelle, «La peinture des donateurs du temple de Maitreya en Mongolie méridionale » Author's own file, not the published version

Please see the published version in Arts Asiatiques 54 (1999), p. 85-102

${ }^{\text {lv }}$ Comme la robe de soie du musée de Mongolie-Intérieure provenant d'une tombe à Dasujixiang

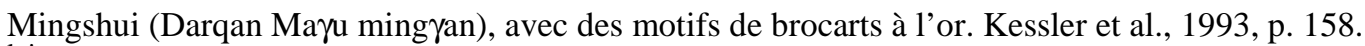

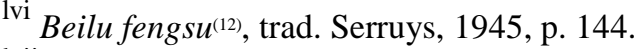

lvii Serruys, 1959, p. 58-59.

lviii Sur un rouleau horizontal joint à une lettre adressée le 17 août 1580 à l'empereur concernant le tribut de chevaux à la cour des Ming, Altan qan porte « un vêtement rouge tissé d'or ». « Sa femme a un costume bleu et rouge brodé d'or », avec des épaulettes, et sans doute deux vêtements superposés. Chavannes, 1896, p. 173-179. Cette lettre écrite en chinois et mongol a été achetée à Pékin par Aleksei M. Pozdneev à un lama du Yonghe gong ${ }^{(39)}$ en 1893. La peinture, qui est certainement de facture chinoise, représente le trajet du tribut mongol de Kökeqota à Pékin ; elle est reproduite en noir et blanc dans l'article de Pozdneev, 1895.

lix Voir Hansen \& Nicolaisen éd., 1993, p. 23.

${ }^{\mathrm{lx}}$ Jin Shen ${ }^{(4)}$, s. d., p. 182.

${ }^{\text {lxi }}$ Voir par exemple Roudova, 1988, fig. 126.

${ }^{\text {lxii }}$ Li Yiyou ${ }^{(4)}$, 1980, p. 183-192 ; Jin Shen ${ }^{(4)}$, 1984c, p. 168-169 ; Cheng Xuguang ${ }^{(5)} \&$ Liu Yibin $^{(6)}, 1984$, p. 180.

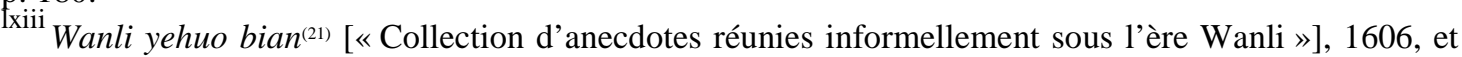
supplément 1619, éd. 1997 [1959], p. 680. Il existe des peintures plus tardives de Jünggen qatun, comme le rouleau peint dans le style chinois, non daté mais portant une inscription, conservé au musée d'Histoire de Chine à Pékin. Au début du siècle étaient encore suspendus dans le temple de l'Impératrice huit rouleaux de brocart représentant une femme mongole dans des décors différents - il s'agissait certainement de Jünggen qatun . Ces rouleaux ont été achetées par un moine «étranger ». Rong Xiang ${ }^{(41)}$, 1979 [1957], p. 227.

lxiv Jin Shen ${ }^{(4)}$, s. d., p. 175.

${ }^{\mathrm{lxv}}$ Erdeni tunumal neretü sudur orusiba [«Chronique nommée Clarté de Joyau »], auteur anonyme,

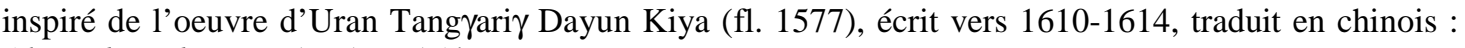
Aletan han zhuan (42), 1991, p. 154.

${ }^{1 x v i}$ Heissig, 1954, p. 10-11, n. 9.

lxvii Erdeni-yin tobci [«Chroniques de joyau »] de Sarang Secen, 1662, trad. Schmidt, 1961 [1829], p. 130, 231 etc. ; colophon du Sili un onol-tu [« Le sot et le sage »], traduit du tibétain, cité par Serruys, 1975 b, p. 236.

lxviii Comme le montre Jin Shen ${ }^{(4)}$ (s. d., p. 177), cela ne peut-être Altan qan (mort en 1582) qui avait une différence d'âge d'une cinquantaine d'années avec elle, ni son deuxième mari, Sengge dügüreng (1538-1585), dont le physique était particulièrement repoussant, et qui avait plus de vingt femmes. Jünggen qatun avait été contrainte de l'épouser et le haïssait.

${ }^{\text {lxix }}$ Jin Shen ${ }^{(4)}$, s. d., p. 177.

${ }^{\text {lxx }}$ Cheng Xuguang ${ }^{(5)} \&$ Liu Yibin ${ }^{(6)}$, 1984, p. 180. C'est également l'opinion de Li Yiyou ${ }^{(40)}$, 1980, p. 183-192.

lxxi Jin Shen ${ }^{(4)}$, 1984c, p. 168-169.

lxxii Elle est appelée « incarnation de la Târâ précieuse » dans plusieurs oeuvres, dont le colophon du Silizun onol-tu, cité par Serruys, 1975a, p. 178-179.

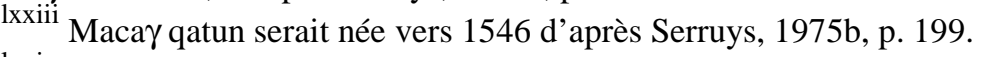

lxxiv Jin Shen ${ }^{(4)}, 1984$ c, p. 169.

${ }^{\text {lxxv }}$ Budasiri, qui meurt en 1597. Elle serait donc veuve en 1606, pourtant elle porte sur la peinture la coiffure d'une femme mariée.

${ }^{\text {lxxvi }}$ Erdeni-yin tobci, éd. et trad. Schmidt, 1961 [1829], p. 261.

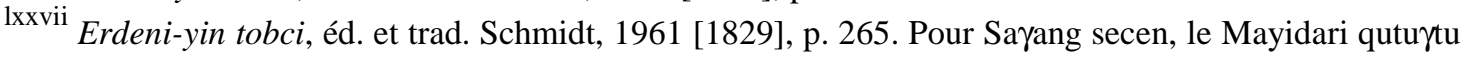

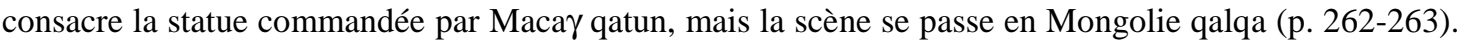
Selon une tradition non orthodoxe, sa réincarnation suivante serait le Jebjündamba quturtu : il est alors confondu avec Târanâtha, moine jonang-pa. 
Charleux, Isabelle, « La peinture des donateurs du temple de Maitreya en Mongolie méridionale » Author's own file, not the published version

Please see the published version in Arts Asiatiques 54 (1999), p. 85-102

${ }^{1 x x v i i i}$ L'ordre des « Vertueux », fondé par le grand théologien tibétain Tsong-kha-pa (1357-1419), insiste sur la discipline monastique et sur la « voie graduelle » de la formation spirituelle.

${ }^{\text {lxxix }}$ Par exemple Râhula, général des planètes et démon des éclipses du panthéon brahmanique, qui joue un rôle important dans le panthéon rnying-ma-pa (pavillon octogonal, Mayidari-yin juu).

${ }^{1 x x x}$ Jin Shen ${ }^{(4)}$, s. d., p. 179 ; Jin Shen ${ }^{(4)}$, 1984c, p. 171.

${ }^{1 x x x i}$ Sous sa forme de lokapâla (Vaiçravana), en sculpture dans le temple aux lokapâla et en peinture sur la partie inférieure des murs ouest et nord du sanctuaire ; sous sa forme de dieu des richesses (Jambhala), en peinture sur la partie inférieure du mur sud du sanctuaire [fig. 10] aux côtés de la déesse des richesses Vasudhârâ [fig. 15], et probablement en sculpture dans le temple qui lui était consacré derrière le Grand temple (Caishen miao $\left.{ }^{(43)}\right)$, c'est-à-dire précisément au centre du Mayidari-yin juu. Pishamen ${ }^{(7)}$ est la seule divinité qui suit l'iconographie chinoise.

lxxxii Cheng Xuguang(5) \& Liu Yibin ${ }^{(6)}, 1984$, p. 180.

lxxxiii Rong Xiang(4), 1979 [1957], p. 227.

lxxxiv Parmi les tenants de cette thèse, citons Wada Sei (Kiyoshi $\left.{ }^{(44)}\right), 1984$ [1959], p. 633.

${ }^{1 x x x v}$ Cette disposition est fixée dans les textes indiens, comme le Mañjuçrîmûlakalpa, traduit en tibétain vers 1060 (Lalou, 1930, p. 15.)

lxxxvi «The Tibetan Pantheon », p. 209 et fig. 5b in Berger, 1995. Ce kesi conservé au Metropolitan Museum of Art de New York représente le mandala de Vajrabhairava (ca. 1328-29).

${ }^{1 x x x v i i}$ En particulier une peinture de la grotte 126 du district 4 à Gu-ge (vers 950-1630) montre la famille royale -deux hommes et deux femmes- assis devant une table d'offrande. En dessous sont disposées trois tables d'offrandes et des serviteurs s'affairent dans la partie inférieure. Jin Weinuo(45), 1991, fig. 177.

lxxxviii Goepper, 1996, fig. 3. Cette peinture de la fin du XI' ou du début du XII siècle fit également l'objet de plusieurs discussions centrées sur les costumes et les coiffures.

lxxxix mchod(-gnas)-yon(-bdag), « objet d'adoration (le lama)-donateur ».

${ }^{\mathrm{xc}} \mathrm{Il}$ en est de même dans la peinture tibétaine. Singer, 1995.

${ }^{\text {xci }}$ Le Grand temple (salle d'assemblée et sanctuaire) et deux autres salles du Mayidari-yin juu, le Grand temple du Caqar blama juu, le sanctuaire et le temple occidental du Yeke juu, le temple occidental du Siregetï juu.

${ }^{\text {xcii }}$ Encore appelées Aerzhai shiku(4), à Aerbasi ${ }^{(47)}$ sumu en Oto $\gamma$, à 130 km au nord-ouest de Wulanzhen ${ }^{(48)}$. Le site est occupé depuis les Wei du Nord (386-534). Wang Dafang(49), Batu Jirigala ${ }^{(50)}$ \& Zhang Wenfang ${ }^{(51)}, 1994$, p. 566-578.

${ }^{\text {xciii }}$ D'après Wang Dafang ${ }^{(49)}$, Batu Jirigala ${ }^{(50)}$ et Zhang Wenfang(51) $(1994$, p. 572), le style des grottes Ming (XVe-XVII ${ }^{e}$ siècles) est semblable à celui des peintures du temple de Maitreya. La fig. 2 de leur article montre deux donateurs principaux, assis, entourés de laïcs et de moines debout.

${ }^{\text {xciv }}$ Charleux, 1998, p. 352-353.

${ }^{\mathrm{xcv}}$ Charleux, 1998, fig. 39, p. 635.

${ }^{\mathrm{xcvi}}$ Les peintures sont partiellement préservées sous forme de panneaux au musée d'Histoire de Kökeqota. 